To appear in The Astrophysical Journal Supplement Series

\title{
A Search for "Dwarf" Seyfert Nuclei. III. Spectroscopic Parameters and Properties of the Host Galaxies
}

\author{
Luis C. Ho \\ Department of Astronomy, University of California, Berkeley, CA 94720-3411 \\ and \\ Harvard-Smithsonian Center for Astrophysics, 60 Garden St., Cambridge, MA 02138円 \\ Alexei V. Filippenko \\ Department of Astronomy, University of California, Berkeley, CA 94720-3411 \\ and \\ Wallace L. W. Sargent \\ Palomar Observatory, 105-24 Caltech, Pasadena, CA 91125
}

\begin{abstract}
We have completed an optical spectroscopic survey of the nuclear regions $(r \lesssim 200$ pc) of a large sample of nearby galaxies. Although the main objectives of the survey are to search for low-luminosity active galactic nuclei and to quantify their luminosity function, the database can be used for a variety of other purposes. This paper presents measurements of the spectroscopic parameters for the 418 emission-line nuclei, along with a compilation of the global properties of all 486 galaxies in the survey. Stellar absorption generally poses a serious obstacle to obtaining accurate measurement of emission lines in nearby galactic nuclei. We describe a procedure for removing the starlight from the observed spectra in an efficient and objective manner. The main parameters of the emission lines (intensity ratios, fluxes, profile widths, equivalent widths) are measured and tabulated, as are several stellar absorption-line and continuum indices useful for studying the stellar population. Using standard nebular diagnostics, we determine the probable ionization mechanisms of the emission-line objects. The resulting spectral classifications provide extensive information on the demographics of emission-line nuclei in the local regions of the universe. This new catalog contains over 200 objects showing spectroscopic evidence for recent star formation and an equally large number of active galactic nuclei, including 46 which show broad $\mathrm{H} \alpha$ emission. These samples will serve as the basis of future studies of nuclear activity in nearby galaxies.
\end{abstract}

\footnotetext{
${ }^{1}$ Present address.
} 
Subject headings: galaxies: active — galaxies: general — galaxies: nuclei — galaxies: Seyfert — nebulae: H II regions — surveys

\section{Introduction}

We have recently completed an extensive optical spectroscopic survey of the nuclei $(r \lesssim 200$ pc) of nearby galaxies. The main goals of the survey are to systematically search for and to investigate the physical properties of low-luminosity, or "dwarf," active galactic nuclei (AGNs), but the rich database can be used for a variety of other applications. In previous papers of this series, we have outlined the scientific objectives and observational parameters in greater detail (Filippenko \& Sargent 1985, hereafter Paper I; Ho, Filippenko, \& Sargent 1995, hereafter Paper II), and several companion papers present various aspects of the analysis (Ho, Filippenko, \& Sargent 1997a, b, c, d; Ho et al. 1997e). In brief, high-quality, long-slit, optical spectra of moderate resolution were obtained of the nucleus of almost every bright galaxy in the northern $\left(\delta>0^{\circ}\right)$ sky. The chosen magnitude limit is $B_{T}=12.5 \mathrm{mag}$, and the objects were selected from the Revised Shapley-Ames Catalog of Bright Galaxies (RSA; Sandage \& Tammann 1981). The spectra were acquired using the Hale $5 \mathrm{~m}$ telescope at Palomar Observatory and cover the regions $\sim 4230-5110$ $\AA$ and $\sim 6210-6860 \AA$, with spectral resolutions of approximately $4 \AA$ and $2.5 \AA$, respectively. Paper II presents a complete spectral atlas of the 486 galaxies contained in the Palomar survey.

The observational distinction between low-luminosity and high-luminosity AGNs is largely arbitrary. Our survey contains mostly nearby galaxies and is designed to select objects on the faint end of the luminosity function. Thus, our study probes much lower luminosities than in other, more conventional AGN samples. The Palomar AGNs have a median (extinction-corrected) $\mathrm{H} \alpha$ luminosity of only $2 \times 10^{39} \mathrm{ergs} \mathrm{s}^{-1}$, and over $85 \%$ of the sources lie below $\mathrm{L}(\mathrm{H} \alpha)=10^{40} \mathrm{ergs} \mathrm{s}^{-1}$ (Ho et al. 1997a). By contrast, the typical Seyfert nucleus in the widely-studied Markarian catalog emits roughly $10^{41} \mathrm{ergs} \mathrm{s}^{-1}$ in $\mathrm{H} \alpha$ (estimated from the compilations of Mazzarella \& Balzano 1986 and Whittle 1992a). Operationally, we will henceforth define "low-luminosity" or "dwarf" AGNs to be those with $\mathrm{L}(\mathrm{H} \alpha) \leq 10^{40} \operatorname{ergs~s}^{-1}$.

This paper focuses on the quantification of spectroscopic parameters from the Palomar survey. The measurements of the emission lines are presented in $\S 2$, where we also discuss in depth our method of starlight subtraction. Section 3 describes our method of spectral classification and the results of applying it to our survey, while section 4 presents the measurements of several stellar absorption-line and continuum indices useful for studying the stellar population. Finally, for easy reference and subsequent analysis, we summarize in $\S 5$ a number of global properties of the host galaxies. 


\section{Emission Lines}

\subsection{Starlight and Continuum Subtraction}

The optical spectra of many emission-line nuclei, particularly those residing in host galaxies of early to intermediate Hubble types (Sbc and earlier), are contaminated heavily, and often dominated, by the stellar component. As late-type giants dominate the integrated light of galaxy bulges, stellar absorption lines affect the strengths of most emission lines of interest. The magnitude of the effect obviously depends on the equivalent widths of the emission lines, but it is generally large in the centers of most "normal" galaxies, where the emission lines are quite weak. Consequently, quantitative studies of most emission-line nuclei depend sensitively on the accuracy with which the stellar absorption lines can be removed.

We adopt a modified version of the technique of "template subtraction" to correct for starlight contamination. The strategy is straightforward: subtract from the spectrum of interest a suitably scaled template spectrum (model) which best represents the continuum and absorption-line strength of the stellar component; the resulting end-product should then be a continuum-subtracted, pure emission-line spectrum. The template model is constructed from a library of galaxy absorption-line spectra. Employing a minimization algorithm, we find the linear combination of individual spectra, which, when suitably normalized and velocity-broadened, best fits the spectral regions in the object of interest that are free of emission lines.

The large number of galaxies in our survey presents us with an extensive collection of absorption-line spectra spanning a wide range of velocity dispersion, metallicity, and stellar population. Since our survey is observationally unbiased with respect to the presence or absence of emission lines, fairly uniform signal-to-noise ratios $(\mathrm{S} / \mathrm{N})$ were achieved for all galaxies. In total, there are 65 galaxies free of visible emission lines; an additional 14 have only very weak emission lines in the red spectrum and almost none in the blue spectrum. The library of 79 templates (Table 1) was ranked according to velocity dispersion and approximate metallicity, the latter judged by the average equivalent widths of three strong Fe I features in the blue spectrum ( $\S 4.2)$. The diversity of the template library can be seen in the sample spectra shown in Figure 1.

The $\chi^{2}$-minimization algorithm was adapted from a code originally developed to study the line-of-sight velocity distributions of early-type galaxies (see Rix \& White 1992 for a full description). First, we corrected all the spectra, both the emission-line objects and the templates, for reddening due to the Galaxy (Burstein \& Heiles 1982, 1984) using the extinction law of Cardelli, Clayton, \& Mathis (1989). This step removes gross continuum shape mismatches between the template and object spectra due to extrinsic dust. Given a list of input template spectra and an initial guess of the velocity dispersion, the program solves for the systemic velocity, the line-broadening function (assumed to be a Gaussian), the relative contributions of the various templates, and the overall continuum shape. In general, a good solution for the absorption-line spectrum does not guarantee an equally good match for the continuum shape, as the latter can 
be affected by dust intrinsic to the galaxies, by residual bandpass calibration errors, and by the non-uniqueness of the continuum shape as predicted by the strength of the stellar absorption lines. The best-fitting model is then subtracted from the original spectrum, yielding a pure emission-line spectrum. We typically chose from our master list one to several candidates whose velocity dispersions, metallicity, and range in stellar populations bracket the probable values in the object of interest. We often iterated this process several times using different combinations of input templates. Each iteration was applied separately to the blue and the red spectra, since the two are not contiguous. We selected the final, best model by inspecting the flatness of the residual spectrum in regions not containing strong emission lines.

Figure 2 illustrates this process for NGC 3596, whose nucleus is undergoing star formation, and for the Seyfert 2 nucleus of NGC 7743. In the case of NGC 3596, the model consists of a combination of the spectrum of NGC 205, a dE5 galaxy dominated by A-type stars, and NGC 4339, an elliptical with a K-giant spectrum. Note that in the original observed spectrum (top), $\mathrm{H} \gamma$, [O III] $\lambda \lambda 4959,5007$, and [O I] $\lambda 6300$ are hardly visible, whereas they can be easily measured after starlight subtraction (bottom). The intensities of both $\mathrm{H} \beta$ and $\mathrm{H} \alpha$ were modified substantially, and the ratio of the doublet [S II] $\lambda \lambda 6716,6731$ lines changed. The effective template for NGC 7743 also makes use of NGC 205 and NGC 4339, but in this case the intermediate-age population contributes substantially less; moreover, the fit improved after including a contribution from NGC 628, an Sc galaxy with a nucleus dominated by A and F stars. Additional examples of starlight subtraction in Figures 3 and 4 depict the range of emission-line intensities encountered in practice. The weakest emission lines we can measure have equivalent widths of $\sim 0.25 \AA$, and we take this to be our detection limit $(3 \sigma)$.

The philosophy behind our empirical method is simple and close in spirit to that of a similar technique often employed in the past (e.g., Costero \& Osterbrock 1977; Koski 1978; Shuder \& Osterbrock 1981; Peimbert \& Torres-Peimbert 1981; Rose \& Searle 1982; Filippenko \& Halpern 1984; Filippenko 1985; Filippenko \& Sargent 1988; Ho, Filippenko, \& Sargent 1993). The templates used in these previous studies, however, were generally derived either from the spectrum of a single, different galaxy with weak or undetectable emission lines or from the spectrum of an off-nuclear position in the same galaxy. Both cases, however, can suffer from significant shortcomings. In the first instance, the absorption-line galaxy chosen as the template may not accurately reflect the stellar content and velocity dispersion of the object in question. Most studies generally invest only limited observing time to the acquisition of template spectra, and the suitability of the choice of templates is not always stated explicitly. In the second case, the off-nuclear spectrum may not be completely free of line emission, and, if there are radial gradients in the stellar population, the off-nuclear light may be a poor representation of the nuclear position. Our implementation of the template-subtraction technique largely overcomes these difficulties. Instead of using a single (and often subjectively determined) spectrum as the template, we use an objective algorithm to find the best combination of spectra to create an "effective" template. The advantages of this modification are obvious. Use of a large basis of input spectra ensures a 
closer match to the true underlying stellar population, while automation of the process minimizes subjective judgement and allows efficient processing of the large database.

Our method of starlight removal can be futher compared with those which generate the best-fitting model for the stellar spectrum from population synthesis (e.g., Keel 1983b). Although physically more rigorous, the appropriateness of the latter approach may be questionable. The available input stellar libraries are drawn mainly from stars with solar metallicity, while galaxy nuclei, especially those in early-type systems, tend to be metal-rich environments (Spinrad \& Taylor 1971). Indeed, for many of his objects, Keel was unable to measure even relatively prominent emission lines such as $\mathrm{H} \beta$. In an effort to circumvent some of the problems encountered in traditional synthesis techniques, Bica and co-workers (Bica \& Alloin 1986; Bica 1988; Storchi-Bergmann, Bica, \& Pastoriza 1990) have compiled a spectral library of star clusters having a range of metallicities.

Finally, a common practice involves fitting the Balmer absorption and emission series simultaneously by assuming that all the absorption lines have the same equivalent width (e.g., McCall, Rybski, \& Shields 1985; Dinerstein \& Shields 1986; Liu \& Kennicutt 1995; Veilleux et al. 1995). Although $\mathrm{H} \delta, \mathrm{H} \gamma$, and $\mathrm{H} \beta$ have roughly comparable equivalent widths under a variety of conditions, $\mathrm{H} \alpha$ appears to show deviations (Díaz 1988). Moreover, the profiles of the Balmer absorption series are pressure broadened, especially for a young to intermediate-age population; failure to account for this in the line-fitting process, as seems to have been the case in the above-mentioned studies, will severely compromise the outcome of the line decomposition. Moreover, in fitting only the Balmer lines, one implicitly assumes that stellar absorption does not affect the other emission lines in the optical window. This, however, is unjustified for old stellar systems: stellar features affect the majority of emission lines to some degree (e.g., Fig. 3 of Ho et al. 1993), and the effect can be severe if the emission lines are weak.

Column 13 of Table 2 lists the template(s) used for each of the 418 emission-line galaxies in the survey. Nonstellar, featureless emission dominates the continuum in several luminous Seyfert galaxies (e.g., NGC 1275, NGC 3227, NGC 4151), and starlight subtraction need not be applied for these. Likewise, the continuum is essentially featureless for a few objects with intense star-forming regions (e.g., IC 10, NGC 784, NGC 1569). For these objects, we fitted the continuum with a low-order spline function and subtracted it prior to line measurement.

\subsection{Measurement of Emission Lines}

After starlight subtraction, the intensity, full width at half maximum (FWHM), and central wavelength were determined for all emission lines detected. The prominent lines include $\mathrm{H} \gamma$, $\mathrm{H} \beta,[\mathrm{O} \mathrm{III}] \lambda \lambda 4959,5007,[\mathrm{O} \mathrm{I}] \lambda \lambda 6300,6363,[\mathrm{~N} \mathrm{II}] \lambda \lambda 6548,6583, \mathrm{H} \alpha$, and [S II] $\lambda \lambda 6716,6731$. In many cases, the lines can be measured straightforwardly by fitting a single Gaussian to each line, with the following exceptions. (1) Profile fitting is unreliable for very noisy data, for lines 
that are affected by large residuals from poor starlight subtraction, or for lines with exceptionally complex structure, such as those showing boxy profiles or multiple velocity components. In these cases, we resorted to summing the line intensity between two endpoints interactively specified. (2) The strength of $\mathrm{H} \beta$ emission in a few objects is either unavailable (e.g., the corresponding pixels were damaged by cosmic rays) or otherwise very unreliable because of severe template mismatch. Since our spectral classification of the nuclei ( $\S 3$ ) depends on $\mathrm{H} \beta$, we simply fixed its strength in these cases based on that of $\mathrm{H} \alpha$ by adopting a theoretical ratio of the two lines, with the implicit assumption that the reddening is negligible. (3) Approximately $10 \%$ of the sample exhibits broad $\mathrm{H} \alpha$ emission and requires more involved line decomposition; this subset of objects is discussed in detail by Ho et al. (1997e). (4) A significant fraction of the galaxies, especially those with active nuclei, have lines with obviously asymmetric shapes and other deviations from a single Gaussian profile. If warranted by the data quality, these cases were fitted with multiple Gaussian components to better characterize the parameters of the line profile.

We placed upper limits on the intensities of undetected, but diagnostically important, emission lines by simulating measurements of the noise level near the expected positions of the lines. The widths of the measurement "windows" were assumed to be comparable to the FWHMs of the lines which are detected in the same object.

Table 2 (cols. 2-8) summarizes the observed (not corrected for reddening) intensities of the main emission lines relative to the intensity of the narrow component of $\mathrm{H} \alpha$; we chose the normalization relative to $\mathrm{H} \alpha$ instead of $\mathrm{H} \beta$ because the former is usually better determined. If the observing conditions were deemed to be approximately photometric (see Paper II), we list the $\mathrm{H} \alpha$ flux (col. 9) and the associated luminosity (col. 10) using the distances adopted in Table 10; nonphotometric measurements are given as lower limits. Column 11 lists the equivalent width of the narrow $\mathrm{H} \alpha$ emission line, obtained by dividing the $\mathrm{H} \alpha$ intensity by the continuum level at $6563 \AA$.

In some spectra, particularly those with sufficiently high $\mathrm{S} / \mathrm{N}$, one or more of the following weaker lines were also measurable, depending on the object: [O III] $\lambda 4363$, He I $\lambda 4471$, He II $\lambda 4686$, [S III] $\lambda 6312,[\mathrm{Fe} \mathrm{X}] \lambda 6374$, and He I $\lambda 6678$. The intensities of these lines, where detected, are summarized in Table 3.

Previous studies of the profiles of narrow emission lines (e.g., Heckman et al. 1981; Feldman et al. 1982; Whittle 1985) generally use [O III] $\lambda 5007$ as the fiducial line, since it is normally the brightest line in the optical spectrum and is usually unaffected by blending with other lines. In our survey, however, we will use [N II] $\lambda 6583$ instead of [O III] $\lambda 5007$ as the fiducial line for line-width measurements, since [N II] lies in the red spectrum, and the S/N and spectral resolution of the red spectra are superior to those of the blue spectra. Despite being partly blended with $\mathrm{H} \alpha$ in some instances, [N II] $\lambda 6583$ is normally the strongest line in the red spectrum next to $\mathrm{H} \alpha$, and, unlike $\mathrm{H} \alpha$, it is not strongly affected by potential uncertainties introduced by imperfect starlight subtraction. The FWHM values of [N II] are given in column 12 of Table 2. The FWHM 
is the simplest, albeit not necessarily the most reliable (e.g., Whittle 1985), indicator of the line width. Nevertheless, we present the FWHM measurements here as a first-order parameterization of the emission-line kinematics; a more extensive analysis of the line profiles is deferred to a later paper. The tabulated line widths were corrected for instrumental resolution by subtracting the instrumental width in quadrature from the observed width: $\mathrm{FWHM}^{2}=\mathrm{FWHM}_{\mathrm{obs}}^{2}-\mathrm{FWHM}_{\text {inst }}^{2}$. FWHM $_{\text {inst }}$ is obtained by fitting a Gaussian profile to a strong, unblended emission line near 6583 $\AA$ in the comparison lamp spectrum taken closest in time to the object of interest The lines in the comparison lamp spectra are very nearly Gaussian in shape. To minimize the possibility of corrupting the final FWHM values from systematic errors in the determination of the instrumental profile, we only apply the correction if $\mathrm{FWHM}_{\text {obs }}$ exceeds $\mathrm{FWHM}_{\text {inst }}$ by at least $50 \%$. Widths smaller than this are reported as upper limits.

\subsection{Reddening Correction}

We adopt the Cardelli et al. (1989) extinction curve to correct the line intensities for reddening due to Galactic dust; the reddening values we assume, taken from Burstein \& Heiles $(1982,1984)$, are listed in column 3 of Table 11 . Next, we use the corrected $\mathrm{H} \alpha / \mathrm{H} \beta$ intensity ratio (Table 4; col. 2) to calculate the internal reddening $\left[E(B-V)_{\text {int }}\right.$; col. 3] due to dust which is either intrinsically associated with the line-emitting gas or lying in its foreground, but belonging to the host galaxy. The Cardelli et al. extinction curve was again assumed. For objects whose spectra resemble $\mathrm{H}$ II regions, we take the intrinsic $\mathrm{H} \alpha / \mathrm{H} \beta$ ratio to be 2.86 , appropriate for case $\mathrm{B}$ recombination in photoionized nebulae with $n_{e}=10^{2} \mathrm{~cm}^{-3}$ and $T_{e}=10^{4} \mathrm{~K}$ (Brocklehurst 1971), whereas for AGNs we adopt an intrinsic ratio of 3.1 (Halpern \& Steiner 1983; Gaskell \& Ferland 1984). Note that in some objects the observed Balmer decrement is significantly less than the theoretical value. These obviously discrepant values, which are usually either lower limits or otherwise have a low quality rating, most likely can be attributed to inaccurate starlight subtraction, especially for $\mathrm{H} \beta$. We assign an internal extinction of zero for these objects.

Table 4 (cols. 4 and 6 through 9) lists five important diagnostic line ratios. They have been corrected for Galactic and internal reddening, although in all cases the correction is nearly negligible because of the close wavelength separation of the lines used in the ratios.

\footnotetext{
${ }^{2}$ The median $\mathrm{FWHM}_{\text {inst }}$ for the entire survey is found to be $2.4 \AA$, somewhat smaller than the nominal value of $2.5 \AA$. For completeness, we note that the median FWHM $_{\text {inst }}$ of the blue spectra near $\lambda=5000 \AA$ is $3.8 \AA$, again slightly better than the nominal resolution of $4 \AA$.
} 


\subsection{Electron Densities}

The electron density, $n_{e}$, can be estimated from the ratio $R([\mathrm{~S}$ II $]) \equiv[\mathrm{S}$ II $] \lambda 6716 /[\mathrm{S}$ II $] \lambda 6731$. $R$ ([S II $]$ ) serves as an effective densitometer (e.g., Osterbrock 1989) so long as $n_{e}$ does not greatly exceed the critical density of $[\mathrm{S} \mathrm{II}]\left(\sim 3 \times 10^{3} \mathrm{~cm}^{-3}\right)$, above which the lines become collisionally de-excited. We used the NEBULAR package implemented within IRAF3 to compute $n_{e}$ (Table 4; col. 5) from $R\left([\mathrm{~S} \mathrm{II}]\right.$ ) (col. 4), assuming $T_{e}=10^{4} \mathrm{~K}$. The software, described by Shaw \& Dufour (1995), assumes that the ground-state electron configuration of common ions such as $\mathrm{S}^{+}$can be approximated by a five-level atom. The atomic data for $\mathrm{S}^{+}$are those published by Cai \& Pradhan (1993).

\subsection{Uncertainties and Error Estimates}

It is generally difficult to evaluate the uncertainty associated with the starlight-subtraction process, which in almost all cases dominates the systematic errors in the line measurements. Hence, formal errors are not assigned to the entries in Tables 2-4. Instead, we employ less quantitative, but nevertheless informative, quality ratings (blank, "b," and "c") to denote the probable reliability of the quoted values. Throughout the tables, entries not followed by a flag (blank rating) are deemed to be of the best quality. We estimate that, on average, the highest accuracy achieved for the line intensities is on the order of $\pm 10 \%-30 \%$, depending on the line. A quality rating of "b" signifies probable errors of $\pm 30 \%-50 \%$, while a rating of "c" pertains to probable errors as large as $\pm 100 \%$. Note that these estimates do not apply to absolute fluxes, which are discussed below. The assignment of the ratings was based on our experience with data of this type, on repeated measurements of objects having multiple observations, and on extensive experimentation with a range of template models.

Being relatively bright in the red region and not strongly affected by stellar absorption, the intensities of [N II] and [S II] can be determined to an accuracy of $\sim 10 \%$ in the best cases. [O I] $\lambda 6300$, athough not crucially dependent on starlight subtraction, is generally much weaker, and it is difficult to achieve errors less than 20\%-30\%. Although [O III] $\lambda 5007$ is usually quite bright and not strongly affected by absorption, the blue spectra of our survey unfortunately have lower sensitivity than the red spectra; nevertheless, accuracies of $10 \%-20 \%$ can be attained for this line. The Balmer series potentially suffers from the most serious uncertainty, whose magnitude becomes progressively larger from $\mathrm{H} \alpha$ to $\mathrm{H} \gamma$. Because of the wavelength coincidence between the emission and absorption components, and because the strength of the absorption component depends sensitively on the age of the stellar population, the intensities of the hydrogen emission lines can vary quite dramatically depending on the choice of template model. Thus, template

\footnotetext{
${ }^{3}$ IRAF is distributed by the National Optical Astronomy Observatories, which are operated by the Association of Universities for Research in Astronomy, Inc., under cooperative agreement with the National Science Foundation.
} 
mismatch can introduce rather large uncertainties in the strengths of the hydrogen emission lines. On the other hand, we believe that we can ascertain the suitability of the chosen template model. Since the hydrogen absorption lines usually have pressure-broadened, Voigt profiles, their wings are much broader than those of the sharper emission component, thus making it possible to judge the appropriateness of the template model: if the template undersubtracts the absorption, a shallow "bowl" is evident beneath the emission line; conversely, if oversubtraction occurs, a broad "hump" would be noticeable. Given that the equivalent widths of the Balmer absorption lines rarely exceed 2-3 $\AA$ (see $\S 4.2$ and Table 9), it is reasonable to assume that emission lines with an equivalent width $\sim 10 \AA$ should have an error of at most $10 \%$ for $\mathrm{H} \alpha$ and perhaps $20 \%$ for $\mathrm{H} \beta$.

Although we provide emission-line fluxes and luminosities (Table 2) and continuum magnitudes ( $\S 4.1$; Table 8 ) for about $80 \%$ of the survey objects, these values do not have high accuracy for at least two reasons. First, the observations were taken through a narrow slit of fixed width $\left(2^{\prime \prime}\right)$. The narrow-line emission in many objects is known to be both spatially extended and inhomogeneous. The amount of light included for each object obviously depends on the specific spatial distribution of the line emission and of the stellar continuum, on the accuracy of centering the object on the slit and subsequent telescope guiding, and on the seeing conditions. Moreover, because of the fixed slit size, the amount of light admitted into the aperture will depend on the distance of the source. Second, we did not attempt to perform rigorous spectrophotometry; the sky conditions were merely monitored by eye periodically through the night. To make a rough estimate of the accuracy of the fluxes, we compared all available measurements for objects which were observed using the same slit width and in more than one epoch, under purportedly "photometric" conditions. Some of the (unpublished) data were collected with the Hale $5 \mathrm{~m}$ telescope and the double spectrograph, but at lower resolution, and others were obtained from Lick Observatory using the Shane $3 \mathrm{~m}$ telescope and the Kast spectrograph (Miller \& Stone 1993), also at lower resolution. The comparison, which could be made for about 30 galaxies, showed that the fluxes are generally consistent to within $\pm 50 \%$ and never deviated by more than a factor of 2. Therefore, we take this to be the range of internal uncertainty associated with each individual measurement, although the uncertainty in our subsequent analysis should be much less because we only consider the ensemble properties of the sample.

The aperture effect, although expected to be present at some level, evidently is not very severe. The distribution of $\mathrm{H} \alpha$ emission-line fluxes shows no obvious trend with source distance (Fig. 5). The only discernible pattern, due to the Malmquist bias, is the lack of points in the lower left corner of the diagram, implying a deficit of nearby $(\lesssim 20 \mathrm{Mpc})$ high-luminosity sources. Nevertheless, for the other reasons mentioned above, we still strongly caution the reader against using any individual measurement for quantitative applications.

Finally, we consider the reliability of the line-width measurements. The merits of using [N II] $\lambda 6583$ as the representative narrow emission line for kinematic measurements were discussed in $\S$ 2.2. The errors associated with the actual measurement of the FWHM should in general be $\lesssim 10 \%$ of the line width. What is potentially more important are possible systematic biases on the line 
widths introduced during the observations. Under good seeing conditions in which the width of the seeing disk is smaller than the width of the slit, and for two sources having identical intrinsic line widths, the observed line width of an unresolved source will be systematically narrower than that of an extended source, provided that the guiding errors do not exceed the slit width. This effect can therefore introduce a systematic bias in the measured line widths depending on the structure of the nuclear regions. However, we anticipate that this source of error will be unimportant in our survey because the combination of guiding errors and seeing was comparable to the slit width. We confirmed our hypothesis by examining the distribution of the seeing conditions (FWHM) recorded in the observation logs (Fig. 6); there are no systematic differences among the various classes of nuclei $(\S 3)$, with the mean and median seeing FWHMs being essentially identical for the four classes. More importantly, within each class, there are no discernible differences in the average FWHM([N II $]$ ) for the objects observed under "good" seeing compared to those observed under "poor" seeing, where we have taken the dividing line to be FWHM $=1$ 1" 5 . The only possible exception is the Seyfert group, where the average FWHM([N II]) $=274$ and $241 \mathrm{~km} \mathrm{~s}^{-1}$ for seeing FWHM $<1$ 1".5 and $\geq 1$ 1".5, respectively. This trend, however, is opposite to that predicted, and, given the small sample size, is probably statistically insignificant.

\section{Spectral Classification}

The optical spectral region contains several emission lines whose intensity ratios can be used to discriminate different sources of ionization. Baldwin, Phillips, \& Terlevich (1981) suggested several two-dimensional, line-intensity ratio diagrams that have since become widely-used diagnostic tools to classify emission-line objects. In these diagrams, nebulae photoionized by hot, young stars (H II regions) can be distinguished from those photoionized by a harder radiation field, such as that of an AGN. The Strömgren sphere of a classical, ionization-bounded H II region makes a fairly sharp transition between the ionized interior and the surrounding neutral medium. Consequently, the partially-ionized transition zone, from which most of the low-ionization forbidden lines (such as [O I], [S II], and to some extent [N II]) are emitted, is quite limited in size. By contrast, an AGN-like ionizing continuum has sufficient high-energy photons to sustain an extensive partially-ionized zone in clouds optically thick to the Lyman continuum, thereby enhancing the strengths of the low-ionization lines relative to those seen in H II regions. A suitable choice of an excitation indicator, such as [O II] $\lambda 3727 /[\mathrm{O}$ III $] \lambda 5007$ or $[\mathrm{O}$ III $] \lambda 5007 / \mathrm{H} \beta$, further delineates the excitation sequence in H II regions and divides AGNs into a high-excitation class (Seyferts) and a low-excitation class [low-ionization nuclear emission-line regions (LINERs); Heckman 1980b].

The separation between the two principal ionization sources (young stars vs. AGN) and between the two AGN excitation classes (Seyferts vs. LINERs) obviously does not have sharp, rigorously-defined boundaries. In practice, however, one must impose somewhat arbitrary, albeit empirically motivated, criteria to establish an internally consistent system of classification. In this study, we use the diagnostic diagrams recommended by Veilleux \& Osterbrock (1987), since 
they employ line-intensity ratios that are relatively insensitive to reddening ([O III $] \lambda 5007 / \mathrm{H} \beta$, [O I] $\lambda 6300 / \mathrm{H} \alpha,[\mathrm{N} \mathrm{II}] \lambda 6583 / \mathrm{H} \alpha$, and [S II] $\lambda \lambda 6716,6731 / \mathrm{H} \alpha)$ and that are contained in the spectral coverage of our survey. For concreteness, we adopt the classification criteria outlined in Table 5; these are very similar to those used by Veilleux \& Osterbrock. In addition to H II nuclei, Seyferts, and LINERs, we also recognize a group of "transition objects," noted by Ho et al. (1993) as emission-line nuclei having [O I] strengths intermediate between those of H II nuclei and LINERs. Ho et al. (1993; see also Ho 1996) proposed that transition objects can be most naturally explained as "normal" LINERs whose integrated spectra are diluted or contaminated by neighboring H II regions. In this interpretation, transition objects should be considered part of the LINER class and should be accounted in AGN statistics (if all LINERs are truly AGNs), although an alternative explanation for these objects based solely on stellar photoionization has also been suggested (Filippenko \& Terlevich 1992; Shields 1992).

As discussed in Ho et al. (1997e), broad H $\alpha$ emission can be discerned in a significant number of objects in our survey. Although the typical luminosity and contrast of the broad component are much smaller than those of "classical" type 1 Seyferts, the widths of the H $\alpha$ line (FWHM $\approx$ 1000-4000 $\mathrm{km} \mathrm{s}^{-1}$ ), especially relative to the forbidden lines, signify the presence of a broad-line region. Although the existence of such "intermediate-type" nuclei has been recognized for a long time (Osterbrock 1977, 1981; Shuder 1980), it is normally thought that weak, broad H $\alpha$ emission is widely found only in Seyfert nuclei. The Palomar survey has demonstrated conclusively that broad $\mathrm{H} \alpha$ exists also in LINERs, and perhaps even in some transition objects. Thus, analogous to the conventional nomenclature of Seyfert nuclei, we propose that the "type 1" and "type 2" designations, along with the intermediate types (1.2, 1.5, 1.8, and 1.9; Osterbrock 1981), be extended to include LINERs and transition objects. We use the criteria of Whittle (1992a) to quantitatively assign numerical values for the type index.

We wish to stress that our classification system is defined by a set of spectroscopic criteria that depends entirely on the line-intensity ratios of several prominent, narrow, optical emission lines, rather than by a combination of spectroscopic, photometric, and morphological characteristics, as has been customary in many older studies. The requirement that a Seyfert nucleus be bright and semistellar in appearance (e.g., de Vaucouleurs \& de Vaucouleurs 1968), for instance, in addition to being subjective, obviously cannot be extended to nuclei of very low luminosities, whose contrast relative to the galaxy bulge becomes the imperceptibly low. Likewise, as pointed out by Phillips, Charles, \& Baldwin (1983), the traditionally accepted definition based on the widths of the emission lines (Weedman 1970, 1977; Balzano \& Weedman 1981; Shuder \& Osterbrock 1981) should be abandoned. The widths of the emission lines in active nuclei correlate strongly with luminosity (Phillips et al. 1983; Whittle 1985, 1992b; Ho 1996; Ho et al. 1997d), and imposing an arbitrary line-width cutoff selects objects by their luminosities. Finally, one should resist the practice of automatically labeling objects as "Seyfert 1s" simply from their having emission lines (e.g., $\mathrm{H} \alpha$ ) arising from a broad-line region (e.g., Paper I). As mentioned above, broad lines also exist in objects with lower ionization levels, and these, according to their narrow-line spectra, 
would be classified as either LINERs or transition objects.

The last column of Table 4 lists the adopted classification for each of the 418 emission-line nuclei, while Figures $7 a-7 c$ show the locations of the objects on the three Veilleux \& Osterbrock (1987) diagnostic diagrams. Some representative spectra of each class, offset from each other for clarity, are plotted in Figures 8-12. We detected emission-line nuclei in $86 \%$ of the survey galaxies. In total, there are $206 \mathrm{H}$ II nuclei, 94 LINERs, 65 transition objects, and 52 Seyferts. These make up, respectively, $49 \%, 23 \%, 16 \%$, and $12 \%$ of the emission-line objects and $42 \%, 19 \%, 13 \%, 11 \%$ of the entire galaxy sample (Ho et al. 1997a discusses the overall statistics of the emission-line nuclei in greater detail). One object (NGC 1003) could not be classified because of the large uncertainties in the line ratios.

It should be emphasized that the classification process is not always unambiguous, for at least three reasons. First, the three conditions involving the low-ionization lines ([O I], [N II], and [S II]) do not always hold simultaneously. This reflects the empirical nature of the diagnostic diagrams as well as the possibility of any one line ratio to be enhanced or depressed with respect to the other two as a result of, for instance, selective abundance variations. Second, substantial measurement uncertainty may be associated with any given line-intensity ratio. Finally, one or more of the line ratios is sometimes only available as an upper limit. Thus, each object must be evaluated individually, taking all of these factors into consideration, before a classification can be assigned to it. In view of the potential selective enhancement of nitrogen in galactic nuclei (e.g., Storchi-Bergmann \& Pastoriza 1989, 1990), less weight is given to the $[\mathrm{N} \mathrm{II}] / \mathrm{H} \alpha$ ratio than to either $[\mathrm{O} \mathrm{I}] / \mathrm{H} \alpha$ or $[\mathrm{S} \mathrm{II}] / \mathrm{H} \alpha$. If a reliable measurement of $[\mathrm{O} \mathrm{I}]$ is available, $[\mathrm{O} \mathrm{I}] / \mathrm{H} \alpha$ is given the largest weight, since, of the three low-ionization lines, it is the most sensitive to the shape of the ionizing spectrum. Where more than one classification may be consistent with the data, both are given, with the more likely one, and the one finally adopted, listed first. Some degree of subjectivity must unavoidably enter into the classification process, although we have tried throughout to be as consistent and unbiased as possible.

We should point out that the definition of LINERs adopted here differs from that originally proposed by Heckman (1980b), which is based exclusively on the relative strengths of [O I], [O II], and [O III]. Nevertheless, inspection of the full optical spectra of LINERs (e.g., Ho et al. 1993) reveals that in practice the two sets of classification criteria generally identify the same objects. This merely reflects that $[\mathrm{O} \mathrm{III}] / \mathrm{H} \beta$ inversely correlates with $[\mathrm{O} \mathrm{II}] /[\mathrm{O} \mathrm{III}]$ for conditions of low excitation (see Fig. 2 in Baldwin et al. 1981). Figure 13 shows the distribution of $[\mathrm{O}$ I $] /[\mathrm{O}$ III] for all objects classified as LINERs in our survey according to the definition adopted here and for which this line ratio is securely known. Clearly, almost all of the objects have $[\mathrm{O} \mathrm{I}] /[\mathrm{O} \mathrm{III}] \gtrsim 0.33$, one of Heckman's criteria.

In general, our classifications should be much better determined than those currently available in the literature, and we recommend the classification given here for overlapping objects. Previous studies of this kind (e.g., Stauffer 1982a, b; Keel 1983a, b; Phillips et al. 1986; Véron-Cetty \& 
Véron 1986; Véron \& Véron-Cetty 1986) often relied exclusively on [N II]/H $\alpha$ for classification: any nucleus having $[\mathrm{N} \mathrm{II}] / \mathrm{H} \alpha$ larger than some limit, usually taken to be $\sim 0.6$, was automatically assumed to be "active." As discussed above, basing the classification on just one line ratio can often be misleading, even if [N II] $/ \mathrm{H} \alpha$ statistically correlates well with [O I] $/ \mathrm{H} \alpha$ and $[\mathrm{S} \mathrm{II}] / \mathrm{H} \alpha$ (Fig. 14). The results are all the more unreliable if starlight subtraction has not been treated carefully or consistently, as was the case in some of the older surveys; undersubtraction of the stellar absorption lines, or failure to remove them altogether, will result in substantial overestimation of $[\mathrm{N} \mathrm{II}] / \mathrm{H} \alpha$. Moreover, some of the above-mentioned studies did not have sufficient wavelength coverage to include $\mathrm{H} \beta$ and [O III], thereby leaving the excitation of the objects unconstrained.

In her analysis of a sample of relatively bright Seyfert 2 nuclei and LINERs, Storchi-Bergmann (1991) showed that the $[\mathrm{N} \mathrm{II}] / \mathrm{H} \alpha$ ratio depends systematically on aperture size or source distance - larger apertures include more extranuclear emission from H II regions, thereby diluting the nuclear spectrum and lowering the observed $[\mathrm{N} \mathrm{II}] / \mathrm{H} \alpha$ ratio. If this effect is severe, it can of course change the spectral classification from that of an AGN to an H II nucleus, and the AGN fraction in a survey such as ours will be underestimated. Since our observations were acquired with a fixed slit size, we know that this distance-dependent effect must be present at some level, and we need to evaluate to what extent our classifications might be influenced by it. Figure 15 plots the distribution of $[\mathrm{N} \mathrm{II}] / \mathrm{H} \alpha$ values as a function of distance; all the emission-line objects are shown together as one group, as each of the four spectral classes separately, and with the three AGN classes summed into one. Within the limited range of distances in our sample, the $[\mathrm{N} \mathrm{II}] / \mathrm{H} \alpha$ ratio exhibits no pronounced variation with distance. Variations in effective aperture size do not adversely affect our spectral classifications in the mean, although, of course, small errors may still affect individual objects.

\section{Stellar Parameters}

Our starlight-subtraction procedure yields, as a by-product, the best-fitting continuum and absorption-line model for each galaxy processed in this manner. These model template spectra, together with the original set of templates, contain useful information pertaining to the stellar component of the nuclei. Because of the high $\mathrm{S} / \mathrm{N}$ of our spectra, even relatively weak stellar absorption lines can be measured accurately. Unfortunately, the limited wavelength coverage of our observing setup excludes several key stellar features (e.g., the blue coverage just misses the $4000 \AA$ break to the left, and Mg I $b \lambda 5175$ and Fe I $\lambda 5270$ to the right); nevertheless, it does include a number of stellar indices that can be used as diagnostics of the stellar population. These include the Balmer lines $(\mathrm{H} \gamma, \mathrm{H} \beta$, and $\mathrm{H} \alpha)$, the $\mathrm{G}$ band at $4300 \AA$, Ca I $\lambda 4455$ and $\mathrm{Ca} \mathrm{I}+\mathrm{Fe} \mathrm{I}$ $\lambda 6495$, Fe I $\lambda \lambda 4383,4531,4668$, and occasionally He II $\lambda 4686$ emission from Wolf-Rayet stars. The shape of the continuum additionally provides some constraints on the stellar content and the reddening.

Our survey is also a rich source of stellar velocity dispersions. Because the full analysis of the 
velocity dispersions is rather lengthy and involved, we defer discussion of these measurements to a separate paper.

\subsection{Measurement of Continuum Indices}

The continuum flux density, $f_{\lambda}$, was determined at three points along the spectrum centered on the rest wavelengths of $\mathrm{H} \gamma(4340 \AA), \mathrm{H} \beta(4861 \AA)$, and $\mathrm{H} \alpha(6563 \AA)$. In order to obtain the continuum values in a consistent, objective manner, the spectra were measured using an automated algorithm. For each of the fiducial wavelengths, the mean intensities of two flanking, relatively line-free regions were determined, and the average of the two resulting values was taken to be the continuum level at the center of the line. Table 6 shows the wavelength ranges of the continuum regions adopted. It is necessary to exclude the region containing the absorption line itself so as not to artificially depress the true continuum level. Moreover, averaging over a sufficiently large wavelength window prevents the final value from being significantly affected by small-scale structure that may be present in the spectra. The continuum measurements, expressed in magnitudes $\left(m_{44}, m_{49}, m_{66}\right)$, are listed in Table 8 for all objects observed under photometric conditions, where $m=-2.5 \log f_{\nu}-48.6$, with $f_{\nu}$ in units of $\operatorname{ergs~s}^{-1} \mathrm{~cm}^{-2} \mathrm{~Hz}^{-1}$ (Oke \& Gunn 1983).

We further use the continuum magnitudes to define three "color" indices: $c(44-49) \equiv m_{44}-m_{49}, c(44-66) \equiv m_{44}-m_{66}$, and $c(49-66) \equiv m_{49}-m_{66}$. Since the blue and red spectra for each galaxy were taken simultaneously, their relative calibration should be reliable and does not depend on sky conditions. Hence, color indices are available for all objects.

Lastly, an absolute magnitude, $M_{44}$, is given for the bluest band using the distances assumed in Table 10 (see $\S 5.2)$.

\subsection{Measurement of Equivalent Widths of Absorption Lines}

The definitions of the nine stellar absorption-line indices extracted from the spectra are given in Table 7 (see also Fig. 16 for a graphic representation of the definitions of the indices), and the results are tabulated in Table 9 . In addition to the three individual iron indices, we also calculated

an average iron index, $\left\langle\mathrm{W}(\mathrm{Fe})>\equiv \frac{1}{3}[\mathrm{~W}(\mathrm{Fe} 4383)+\mathrm{W}(\mathrm{Fe} 4531)+\mathrm{W}(\mathrm{Fe} 4668)]\right.$, which should serve as a more robust indicator of the iron strength.

The measurement of equivalent widths, which requires determination of the flux of a spectral feature and its associated continuum level, can be affected by systematic errors depending on the $\mathrm{S} / \mathrm{N}$ of the data, the strength of the feature, and the velocity dispersion. If the feature of interest is weak or the data particularly noisy, for example, marking the endpoints of the feature and the 
appropriate continuum level can sometimes be ambiguous if done manually. To ensure internal consistency in the data set, the equivalent widths were measured noninteractively. Briefly, for each of the indices, the fluxes of three wavelength regions in the rest frame of the object were determined: one region corresponds to the limits of the absorption feature of interest, while two regions on either side define two continuum levels (Table 7). Interpolation of the two flanking continuum values gives the continuum level underneath the spectral feature, and the ratio of the integrated flux of the feature to the continuum flux yields the equivalent width.

\section{Compilation of Host Galaxy Properties}

Subsequent papers in this series will examine possible connections between the spectroscopic characteristics of the nuclei and the more global properties of the host galaxies. In preparation for this analysis, we provide here a compilation of a number of host galaxy parameters (Tables 10 and 11). We follow closely the format and notation of Whittle (1992a), who has recently provided a comprehensive summary of the global properties of bright Seyfert galaxies, although we also list several quantities not given by Whittle. We have relied heavily on the Third Reference Catalogue of Bright Galaxies (RC3; de Vaucouleurs et al. 1991) for much of the data because this catalog has paid considerable attention to synthesizing data from a variety of sources into a single system. Since we will be primarily interested in comparative analysis, the most important consideration is to have at hand a consistent, homogeneous database. A short description of each parameter tabulated is given below.

\subsection{Morphological Type}

We list for each galaxy the mean revised morphological type and the mean numerical Hubble type index $(\mathrm{T})$, as given in the RC3. The morphological classification of the RC3 parallels the system in the first two editions of the catalog, which itself is based on the principles outlined by de Vaucouleurs $(1959,1963)$. In this system, $\mathrm{T}=-6$ to -4 correspond to ellipticals (E), $\mathrm{T}=-3$ to -1 to lenticulars (S0), $\mathrm{T}=0$ to 9 to spirals $(\mathrm{S} 0 / \mathrm{a}-\mathrm{Sm})$, and $\mathrm{T}=10$ to Magellanic irregulars (Im). $\mathrm{T}$ = 90 designates non-Magellanic irregulars or "amorphous" galaxies (I0; Sandage \& Brucato 1979), and $\mathrm{T}=99$ imples a "peculiar" morphology that cannot be fitted into any of the above categories. Among spirals, those that are strongly or weakly barred are designated by the family symbols "B" and "AB," respectively, and nonbarred members by "A". Note that the morphological types given in Paper II were taken from the catalog of nearby galaxies of Kraan-Korteweg (1986), which relies heavily on the RSA. In future analyses, we will use the morphological types from the RC3 as listed in Table 10, instead of those from the RSA given in Paper II, largely because the RC3 system recognizes intermediate bar types (AB), but also because it is more current.

The distribution of Hubble types for the survey sample is summarized in Table 13 and in 
Figure 17. It is apparent that the sample contains virtually every morphological type, and hence provides an excellent representation of the general galaxy population. Since the objects were drawn from a magnitude-limited catalog, however, our sample certainly is not immune to the usual underrepresentation of low surface brightness systems. Ellipticals comprise $12 \%$ of the sample, while lenticulars, spirals, irregulars, and peculiar systems make up 18\%,65\%, 3\%, and $1.5 \%$, respectively. Barred galaxies (AB and B) contribute $56 \%$ to the disk systems (S0-Im) and $59 \%$ of the spiral galaxies in the sample, roughly comparable to the overall representation in the field galaxy population (50\%-60\%; Sellwood \& Wilkinson 1993).

\subsection{Distances}

The majority of the objects in our survey (90\%) overlap with the nearby galaxy catalog of Tully (1988), who derived distances for galaxies closer than $40 \mathrm{Mpc}$ based on the Virgo infall model of Tully \& Shaya (1984). This model assumes an infall velocity of $300 \mathrm{~km} \mathrm{~s}^{-1}$ for the Local Group, $H_{0}=75 \mathrm{~km} \mathrm{~s}^{-1} \mathrm{Mpc}^{-1}$, and a Virgo distance of $16.8 \mathrm{Mpc}$. Although some of the galaxy distances have been slightly modified in the recent literature, we chose to adopt Tully's distances for convenience and for internal consistency. For galaxies farther than $40 \mathrm{Mpc}$, we obtained distances simply from their systemic velocities and $H_{o}=75 \mathrm{~km} \mathrm{~s}^{-1} \mathrm{Mpc}^{-1}$. The distribution of distances in our survey (Fig. 18) has a median value of $17.9 \mathrm{Mpc}$, a mean of 22.8 $\mathrm{Mpc}$, and a standard deviation of $14.9 \mathrm{Mpc}$. The aperture used in the spectral extractions $\left(2^{\prime \prime} \times\right.$ $\left.4^{\prime \prime}\right)$ corresponds to a projected linear size of $180 \mathrm{pc} \times 360 \mathrm{pc}$ for the median distance. The radial velocities of the galaxy nuclei, which are available from our data, will be presented elsewhere along with additional kinematic data.

\subsection{Radial Velocities and Rotational Velocities}

The heliocentric radial velocities, $v_{\mathrm{h}}$, are listed in column 4 of Table 10; these were taken from the RC3, after converting the values given therein in the frame of the Galactic Standard of Rest to the frame of the Sun. They represent the weighted average of the best available redshifts measured through 21-cm neutral hydrogen and optical observations. The $\mathrm{H}$ I redshifts for the Palomar sample have typical uncertainties of $\sim 6 \mathrm{~km} \mathrm{~s}^{-1}$, while the optical measurements have somewhat larger ones, generally $\sim 30 \mathrm{~km} \mathrm{~s}^{-1}$. A histogram of $v_{\mathrm{h}}$ is shown in Figure 19.

The amplitude of the rotation curve of a spiral galaxy provides a measure of its gravitational potential, and hence its total mass. Previous studies of Seyfert galaxies successfully employed the rotational amplitude to assess the role of the bulge in influencing the nuclear activity (e.g., Whittle 1992b), and we plan to do the same for the objects in this survey. Although our spectra were acquired in long-slit mode, the spectrograph was usually not rotated to the photometric major axis of each galaxy, but rather to the parallactic angle to minimize light losses due to 
atmospheric dispersion (see Paper II). Hence, only some of the resulting rotation curves (to be published elsewhere) can be used to derive reliable rotation amplitudes. Fortunately, it has been demonstrated (e.g., Rubin, Ford, \& Thonnard 1978; Whittle 1992a) that the maximum rotational velocity inferred from the width of the integrated H I emission profile of spiral galaxies provides an excellent substitute for the rotational amplitude obtained through direct analysis of an optical rotation curve. Whittle (1992a) showed that W20, the width of the H I profile measured at $20 \%$ of the peak height, correlates extremely well with $\Delta V_{\text {rot }}$, the amplitude of the optical rotation curve, and that the correlation apparently holds for both normal and active galaxies. Accordingly, we have taken the available W20 measurements from the RC3 for our survey objects (largely based on the $\mathrm{H}$ I database of Bottinelli et al. 1990), and these are tabulated as $\Delta \mathrm{V}_{\mathrm{HI}}$ in Table 10. Since the rotational velocities are seen in projection along the line of sight, the true, inclination-corrected rotational amplitudes $\Delta \mathrm{V}_{\text {rot }}^{c}=\Delta \mathrm{V}_{\mathrm{HI}} / \sin i$ are also listed for all the disk systems, where we have used the inclinations given in Table 11 (see $\S 5.8$ ). To ensure that the deprojection correction factor is not excessively large, inclinations less than $30^{\circ}$ were set to $30^{\circ}$.

\subsection{H I Content}

It will be of interest to search for possible connections between the presence and type of nuclear activity and the overall gaseous content of the host galaxies, as some relationship may be found based on the expectation that the availability of interstellar matter should affect the fueling rate of the nuclear regions. Since the RC3 conveniently lists H I fluxes for the majority of the non-elliptical galaxies in our survey, we summarize the H I masses in Table 10, normalized to the "corrected" $B$-band luminosity (see $\S 5.6$ ) of each object, $M_{\mathrm{HI}} / L_{B}^{0}$, where in converting magnitudes to luminosities a solar absolute magnitude of $M_{B}=5.52$ was used. The calculation of the $\mathrm{H}$ I mass from the observed $\mathrm{H}$ I fluxes is explained in the RC3, and, in the present compilation, we simply adopted the fluxes given in the RC3, which account for self-absorption of the 21-cm line. Note that the quantity $M_{\mathrm{HI}} / L_{B}^{0}$ is independent of distance.

\subsection{Galaxy Environment}

That tidal perturbations from neighboring galaxies can trigger the onset of nuclear activity as well as enhance its strength has been documented in numerous studies (e.g., Shlosman 1994 and references therein). For each galaxy, we attempt to gauge the gravitational influence of its nearest neighbors using two estimators. First, we make use of the local galaxy density, $\rho_{\text {gal }}$, defined by Tully (1988) as the density of all galaxies brighter than $M_{B}=-16$ mag in the vicinity of the object of interest. A second, more straightforward approach that has proven to be useful in the past (e.g., Stauffer 1982b) is to measure the separation between a given galaxy and that of its nearest, sizable neighbor. A reasonable strategy might be to locate a neighbor meeting a relative magnitude criterion $(\Delta m)$, as well as a relative velocity criterion $(\Delta v)$. The former selects neighbors falling 
within a given luminosity ratio, which loosely translates into a mass ratio if the luminosity is not abnormally enhanced or depressed, whereas the latter increases the probability that the pair is actually physically associated. We chose $\Delta m \leq 1.5 \mathrm{mag}$ (factor of 4 in luminosity) and $\Delta v= \pm 500 \mathrm{~km} \mathrm{~s}^{-1}$, and we performed an automated search using the NASA/IPAC Extragalactic Database (NED) to measure $\theta_{p}$, the projected angular separation between the primary and the secondary galaxy, measured in units of the isophotal angular diameter of the primary, $D_{25}$ (Table 11, col. 11). The search radius was limited to $300^{\prime}$, the maximum value allowed by the NED search routine; beyond this limit the galaxy can be safely considered to be isolated.

Finally, we consider the environment of each galaxy in a larger context, namely its group and cluster membership. Garcia (1993) recently analyzed the group affiliation for a large sample of nearby galaxies, whose selection criteria encompasses those of the Palomar survey. More than $66 \%(323 / 486)$ of our objects can be assigned to specific groups, and they are identified in Table 10 using the group designation of Garcia. Those objects belonging to the Virgo Cluster or its southern extension (Tully 1987) are additionally noted.

\subsection{Magnitudes and Colors}

The total (asymptotic) $B$-band magnitudes, as observed $\left(B_{T}\right)$ and after correcting for extinction and redshift $\left(B_{T}^{0}\right)$, were taken from the RC3 and are listed in Table 11. The two dominant terms in the magnitude correction are due to internal extinction $\left(A_{i}\right)$, which depends on inclination and is assumed to be significant only for spirals (see RC3), and extinction from the Galaxy, $A_{g}$, which was estimated principally from the H I maps of Burstein \& Heiles (1982, 1984). Because of the proximity of our sample, the redshift $(K)$ correction in almost all cases amounts to less than $0.01 \mathrm{mag}$. The absolute magnitudes, $M_{B_{T}}^{0}=B_{T}^{0}-5 \log (d / 10)[d$ in pc], are derived from the apparent magnitudes and assumed distances. The distributions of $B_{T}$ and $M_{B_{T}}^{0}$ are shown in Figures 20 and 21, respectively.

We also present in Table 11 two broad-band optical colors taken from the RC3, $(U-B)_{T}^{0}$ and $(B-V)_{T}^{0}$, integrated over the galaxy and corrected for the effects of extinction and redshift

as described above. These quantities will be of use for examining global trends in the star-forming properties of the host galaxies.

\subsection{Bulge Luminosities}

The mass of the galactic bulge or spheroidal component most likely exerts the strongest impact on nuclear activity, as the central engine undoubtedly is coupled to the depth of the nuclear gravitational potential. To first order, such an association is already apparent in that AGNs preferentially reside in early-type hosts (Heckman 1980a; Keel 1983b; Terlevich, Melnick, \& Moles 1987; Ho et al. 1997a), in that the kinematics of the line-emitting gas reflect principally 
the velocities of the bulge stars (Nelson \& Whittle 1996), and in the preliminary indication that more massive black holes appear to live in more luminous galaxies (Kormendy \& Richstone 1995). While an explicit bulge-disk decomposition would certainly be desirable, at the moment detailed surface photometry is unavailable for our sample as a whole. We follow Whittle (1992a) and use an average, type-dependent relation to estimate the bulge luminosity from the total galaxy luminosity. Simien \& de Vaucouleurs (1986) give the following empirical relation to estimate the contribution of the disk to the total luminosity of a disk galaxy with $-3 \leq \mathrm{T} \leq 7: \Delta m_{\text {bul }}=$ $0.324(\mathrm{~T}+5)-0.054(\mathrm{~T}+5)^{2}+0.0047(\mathrm{~T}+5)^{3}$. This correction is zero for ellipticals $(\mathrm{T}=-6$ to -4 ), which by definition have no disks, and it equals 1.02 mag for $\mathrm{T}=+1$ (Sa) and $2.54 \mathrm{mag}$ for $\mathrm{T}=+5(\mathrm{Sc})$. In this study, we assume that the empirical relation can be extended to $\mathrm{T}=9$; for $\mathrm{T}>9, \Delta m_{\text {bul }}$ is undefined. The bulge absolute magnitudes, $M_{B}(\mathrm{bul})=M_{B_{T}}^{0}+\Delta m_{\text {bul }}$, are listed in Table 11 and shown in histogram form in Figure 22.

\subsection{Inclinations and Isophotal Diameters}

It is instructive to examine the distribution of galaxy inclination angles to assess possible selection effects. Figure 23 shows the distribution of cosine $i$ for all sample disk galaxies for which the RC3 lists an isophotal axial ratio, $R_{25}=\log (a / b)$, where the major and minor axis diameters, $a$ and $b$, are measured at a $B$ surface brightness level of $25 \mathrm{mag} \operatorname{arcsec}^{-2}$. The inclination then follows from $\sin ^{2} i=\left[1-(b / a)^{2}\right] / 0.96$ (Hubble 1926). The distribution of cosine $i$ should be flat for an unbiased sample with random orientations. Our sample evidently is not completely free from inclination bias, as judged by the increasing deficit of objects for $i \gtrsim 70^{\circ}$; the departure from a uniform distribution is highly significant according to a Kolmogorov-Smirnov test (Press et al. 1986). This behavior, however, is to be expected in a magnitude-limited sample, since internal absorption tends to shift edge-on disk systems above the limiting magnitude (e.g., Burstein, Haynes, \& Faber 1991; Maiolino \& Rieke 1995).

We also extracted from the RC3 the isophotal major axis diameters (measured at $\mu_{B}=25$ mag $\left.\operatorname{arcsec}^{-2}\right)$, both as observed $\left(D_{25}\right)$ and corrected to face-on orientation $\left(D_{25}^{0}\right)$. This parameter will be used as a measure of the linear scale of the systems.

\subsection{Far-infrared Properties}

Finally, we collected the best available far-IR measurements of the host galaxies made by the Infrared Astronomical Satellite (IRAS). These data will be used to analyze the global star-formation properties of the galaxies. The total flux densities $\left(S_{12}, S_{25}, S_{60}\right.$, and $\left.S_{100}\right)$ and the associated errors $(\sigma)$ of the four bands at 12, 25, 60, and $100 \mu \mathrm{m}$, all in units of Janskys, are given in columns 2 through 9 of Table 12. The parameter FIR (col. 10), defined as $1.26 \times 10^{-14}\left(2.58 S_{60}+S_{100}\right) \mathrm{W} \mathrm{m}^{-2}$, approximates well the total flux between 42.5 and $122.5 \mu \mathrm{m}$ 
(Rice et al. 1988; Helou et al. 1988). For convenience, we also tabulated the the ratios of the flux densities of the four bands, or the far-IR colors: $S_{12} / S_{25}, S_{25} / S_{60}$, and $S_{60} / S_{100}$ (cols. 11-13).

Many catalogs of IRAS data exist in the literature. We have strived to adopt the largest, most homogeneous, and most recent compilations available. Flux densities for galaxies with diameters larger than $8^{\prime}$ were taken from Rice et al. (1988), and the remaining ones were chosen, in decreasing order of preference, from Helou et al. (1988), Soifer et al. (1989), Knapp et al. (1989), Rush, Malkan, \& Spinoglio (1993), and Moshir et al. (1990), the latter as given in NED. Additional studies (col. 14) were consulted for a few objects not listed in the above primary sources. (The published version of the Rush et al. catalog did not list uncertainties for the flux densities. B. Rush kindly provided the original data files from which we extracted the uncertainty measurements.)

\section{Summary}

In an effort to search for and to study the physical properties of low-luminosity AGNs, we have completed an optical spectroscopic survey of the nuclear regions of a large sample of nearby galaxies. The survey is useful for many other purposes in addition to the original objectives. This paper describes in detail our procedure of measuring various spectroscopic parameters to characterize the emission lines, the absorption lines, and the stellar continuum. From analysis of the emission-line intensity ratios, spectral classifications are presented for a total of 418 emission-line nuclei. We discuss in depth our procedure for starlight subtraction, as this is a crucial step for accurate measurement of the relatively weak emission lines in these nuclei. Finally, we provide a summary of global galaxy properties compiled from various published catalogs. This information will be used extensively in other papers in this series, where the scientific analysis will be presented.

The research of L. C. H. is currently funded by a postdoctoral fellowship from the HarvardSmithsonian Center for Astrophysics. Financial support for this work was provided by NSF grants AST-8957063 and AST-9221365, as well as by NASA grants AR-5291-93A and AR-5792-94A from the Space Telescope Science Institute (operated by AURA, Inc., under NASA contract NAS5-26555). We thank Hans-Walter Rix for generously providing the code used for the starlight subtraction and Chien Peng for developing the software used for the line-profile fitting. Brian Rush gave valuable advice concerning IRAS data and sent us an electronic version of the extended $12 \mu \mathrm{m}$ galaxy catalog. Anne Manuelle Garcia furnished an electronic file of her catalog of galaxy groups. Chris McKee and Hy Spinrad gave a critical reading of an earlier draft of the manuscript, and helpful input during the final revision of the paper was provided by Bill Keel, Kim McLeod, Roberto Maiolino, and an anonymous referee. Throughout the course of this work, L. C. H. has benefited from discourse with Aaron Barth and Tom Matheson, and well as from spirited consumption of caffeine with Marianne Vestergaard. We made extensive use of the NASA/IPAC 
Extragalactic Database (NED) which is operated by the Jet Propulsion Laboratory, California Institute of Technology, under contract with the National Aeronautics and Space Administration. 


\section{A. Notes on Individual Objects}

Here we record special difficulties, peculiarities, and other miscellaneous notes of interest encountered during the starlight subtraction or in subsequent steps of the analysis.

$N G C$ 278. - Starlight subtraction in the blue spectrum is uncertain. The $\mathrm{H} \beta$ and $\mathrm{H} \gamma$ absorption lines could not be completely removed.

$N G C$ 428. - Spectral classification is very uncertain.

$N G C 474$. - Spectral classification is very uncertain.

$N G C$ 488. - The emission lines have double peaks separated by $\sim 200 \mathrm{~km} \mathrm{~s}^{-1}$.

$N G C 521 .-[\mathrm{O} \mathrm{III}] \lambda 5007$ is very poorly measured.

$N G C$ 1073. - Starlight subtraction in the blue spectrum is uncertain. The $\mathrm{H} \beta$ and $\mathrm{H} \gamma$ absorption lines could not be completely removed.

NGC 1560. - [O I] $\lambda 6300$ is corrupted by emission from the night sky.

NGC 2366. - The spectrum is corrupted by contamination from a superposed $\mathrm{M}$ star, and the object is discarded from the sample.

$N G C$ 2832. - Starlight subtraction very difficult. Spectral classification highly uncertain.

NGC 3034. - The continuum shape of the red spectrum was improperly calibrated, although this does not affect the measurement of the emission lines.

NGC 3077. - The $\mathrm{H} \beta$ and $\mathrm{H} \gamma$ absorption lines could not be completely removed during starlight subtraction.

NGC 3379. - Starlight subtraction very difficult. We could not obtain a reasonable value for $\mathrm{H} \beta$ emission and resorted to constraining it with $\mathrm{H} \alpha$.

NGC 3608. - Starlight subtraction very difficult. We could not obtain a reasonable value for $\mathrm{H} \beta$ emission and resorted to constraining it with $\mathrm{H} \alpha$.

NGC 3646. - The emission lines have a double-peaked profile.

NGC 3665. - The emission lines have a double-peaked profile.

NGC 3884. - [S II] $\lambda 6731$ corrupted.

$N G C$ 3941. - $\mathrm{H} \beta$ strength uncertain by factor $\sim 2$. Classification could change from Seyfert 2 to LINER 2.

NGC 4145. - The spectrum is partly contaminated by an M star. Although we could not remove the stellar component, we were able to estimate the strengths of several emission lines. 
$N G C$ 4220. - $\mathrm{H} \beta$ may be higher than quoted by a factor of $\sim 2$, but this will not change the spectral classification.

NGC 4278. - The [O III] $\lambda 4363$ line quoted in Table 3 may be partly contaminated by residuals from $\mathrm{H} \gamma$.

$N G C$ 4281. - The emission lines are very noisy and have complicated line profiles. We summed several of the key emission lines by manual integration.

$N G C$ 4350. - Starlight subtraction very difficult. We could not obtain a reasonable value for $\mathrm{H} \beta$ emission and resorted to constraining it with $\mathrm{H} \alpha$. The [S II] lines were summed as one complex and then divided into a ratio of 1.5 to 1 for [S II] $\lambda 6716$ and [S II] $\lambda 6731$, respectively.

$N G C$ 4414. - $\mathrm{H} \alpha$ emission corrupted; estimated based on poorly constrained strength of $\mathrm{H} \beta$.

$N G C$ 4472. - Starlight subtraction very difficult. $\mathrm{H} \beta$ emission strength obtained from $\mathrm{H} \alpha$, which itself is very uncertain. The [S II] lines were summed as one complex and then divided into a ratio of 1.5 to 1 for [S II] $\lambda 6716$ and [S II] $\lambda 6731$, respectively.

$N G C$ 4490. - The stellar population is very young, and the Balmer absorption lines are most likely undercorrected. The $\mathrm{H} \alpha / \mathrm{H} \beta$ ratio should be regarded as an upper limit.

$N G C$ 4579. - $\mathrm{H} \beta$ was damaged by a cosmic ray hit; its intensity was scaled from that of $\mathrm{H} \alpha$ emission.

$N G C$ 5322. - Starlight subtraction was very difficult and the choice of template ambiguous. The final line intensities represent the average of 5 independent trials. The two lines of [S II] could not be measured individually; we summed the blend and divided the intensity equally between the two lines, as the emission complex looks relatively flat-topped, indicating that the two lines probably contribute in equal proportion.

NGC 5631. - The Balmer emission lines, especially $\mathrm{H} \beta$, are somewhat uncertain, but in this case the spectral classification should not be affected.

NGC 5656. - The Balmer emission lines are poorly determined. [S II] $\lambda 6731$ is partly corrupted and therefore not reliable.

$N G C 5858$. - The spectrum is very noisy, and the line profiles are quite complex. The line strengths in the red were approximated by eye, and the ratio of the [S II] lines is not meaningful.

$N G C$ 5982. - Starlight subtraction extremely difficult; line intensities and spectral classification uncertain.

$N G C$ 6482. - The blue spectrum shown in Figure 94 of Paper II, which was obtained at Lick Observatory, cannot be that of NGC 6482. The velocity dispersion of the absorption lines in the blue spectrum is inconsistent with that of the red spectrum, and in any event appears to be too small for a giant elliptical galaxy like NGC 6482. The blue spectrum was therefore discarded, and 
we have no information on the excitation of the emission lines. Judging by the strength of [N II] and [S II] compared to $\mathrm{H} \alpha$ and by the relative weakness of [O I], we estimate that the nucleus is probably either a transition object or a Seyfert 2.

NGC 6501. - Starlight subtraction extremely difficult; line intensities and spectral classification uncertain.

NGC 6702. - Starlight subtraction extremely difficult. 


\section{References}

Baldwin, J. A., Phillips, M. M., \& Terlevich, R. 1981, PASP, 93, 5

Balzano, V. A., \& Weedman, D. W. 1981, ApJ, 243, 756

Bica, E. 1988, A\&A, 195, 76

Bica, E., \& Alloin, D. 1986, A\&A, 162, 21

Bottinelli, L., Gouguenheim, L., Fouqué, P., \& Paturel, G. 1990, A\&AS, 82, 391

Brocklehurst, M. 1971, MNRAS, 153, 471

Burstein, D., Haynes, M. P., \& Faber, S. M. 1991, Nature, 353, 515

Burstein, D., \& Heiles, C. 1982, AJ, 87, 1165

Burstein, D., \& Heiles, C. 1984, ApJS, 54, 33

Bushouse, H. A., Lamb, S. A., \& Werner, M. W. 1988, ApJ, 335, 74

Cai, W., \& Pradhan, A. K. 1993, ApJS, 88, 329

Cardelli, J. A., Clayton, G. C., \& Mathis, J. S. 1989, ApJ, 345, 245

Costero, R., \& Osterbrock, D. E. 1977, ApJ, 211, 675

de Vaucouleurs, G. 1959, Handbuch der Physik, 53, 275

de Vaucouleurs, G. 1963, ApJS, 8, 31

de Vaucouleurs, G., \& de Vaucouleurs, A. 1968, AJ, 73, 858

de Vaucouleurs, G., de Vaucouleurs, A., Corwin, H. G., Jr., Buta, R. J., Paturel, G., \& Fouqué, R. 1991, Third Reference Catalogue of Bright Galaxies (New York: Springer) (RC3)

Díaz, A. I. 1988, MNRAS, 231, 57

Dinerstein, H. L., \& Shields, G. A. 1986, ApJ, 311, 45

Feldman, F. R., Weedman, D. W., Balzano, V. A., \& Ramsey, L. W. 1982, ApJ, 256, 427

Filippenko, A. V. 1985, ApJ, 289, 475

Filippenko, A.V., \& Halpern, J. P. 1984, ApJ, 285, 458

Filippenko, A. V., \& Sargent, W. L. W. 1985, ApJS, 57, 503 (Paper I)

Filippenko, A. V., \& Sargent, W. L. W. 1988, ApJ, 324, 134

Filippenko, A. V., \& Terlevich, R. 1992, ApJ, 397, L79

Garcia, A. M. 1993, A\&AS, 100, 47

Gaskell, C. M., \& Ferland, G. J. 1984, PASP, 96, 393

Halpern, J. P., \& Steiner, J. E. 1983, ApJ, 269, L37

Heckman, T. M. 1980a, A\&A, 87, 142

Heckman, T. M. 1980b, A\&A, 87, 152

Heckman, T. M., Miley, G. K., van Breugel, W. J. M., \& Butcher, H. R. 1981, ApJ, 247, 403

Held, E. V., \& Mould, J. R. 1994, AJ, 107, 1307

Helou, G., Khan, I. R., Malek, L., \& Boehmer, L. 1988, ApJS, 68, 151 
Ho, L. C. 1996, in The Physics of LINERs in View of Recent Observations, ed. M. Eracleous, et al. (San Francisco: ASP), 103

Ho, L. C., Filippenko, A. V., \& Sargent, W. L. W. 1993, ApJ, 417, 63

Ho, L. C., Filippenko, A. V., \& Sargent, W. L. W. 1995, ApJS, 98, 477 (Paper II)

Ho, L. C., Filippenko, A. V., \& Sargent, W. L. W. 1997a, ApJ, in press

Ho, L. C., Filippenko, A. V., \& Sargent, W. L. W. 1997b, ApJ, in press

Ho, L. C., Filippenko, A. V., \& Sargent, W. L. W. 1997c, ApJ, in press

Ho, L. C., Filippenko, A. V., \& Sargent, W. L. W. 1997d, in preparation

Ho, L. C., Filippenko, A. V., Sargent, W. L. W., \& Peng, C. Y. 1997e, ApJS, in press

Hubble, E. 1926, ApJ, 64, 321

Keel, W. C. 1983a, ApJS, 52, 229

Keel, W. C. 1983b, ApJ, 269, 466

Knapp, G. R., Guhathakurta, P., Kim, D.-W., \& Jura, M. 1989, ApJS, 70, 329

Kormendy, J., \& Richstone, D. O. 1995, ARA\&A, 33, 581

Koski, A. T. 1978, ApJ, 223, 56

Kraan-Korteweg, R. C. 1986, A\&AS, 66, 255

Liu, C. T., \& Kennicutt, R. C. 1995, ApJS, 100, 325

Maiolino, R., \& Rieke, G. H. 1995, ApJ, 454, 95

Mazzarella, J. M., \& Balzano, V. A. 1986, ApJS, 62, 751

McCall, M. L., Rybski, P. M., \& Shields, G. A. 1985, ApJS, 57, 1

Melisse, J. P. M., \& Israel, F. P. 1994, A\&AS, 103, 391

Miller, J. S., \& Stone, R. P. S. 1993, Lick Obs. Tech. Rep., No. 66

Moshir, M., et al. 1990, IRAS Catalogs, The Faint Source Catalog, Version 2.0 (Pasadena, JPL)

Nelson, C. H., \& Whittle, M. 1996, ApJ, 465, 96

Oke, J. B., \& Gunn, J. E. 1983, ApJ, 266, 713

Osterbrock, D. E. 1977, ApJ, 215, 733

Osterbrock, D. E. 1981, ApJ, 249, 462

Osterbrock, D. E. 1989, Astrophysics of Gaseous Nebulae and Active Galactic Nuclei (Mill Valley: Univ. Science Books)

Peimbert, M., \& Torres-Peimbert, S. 1981, ApJ, 245, 845

Phillips, M. M., Charles, P. A., \& Baldwin, J. A. 1983, ApJ, 266, 485

Phillips, M. M., Jenkins, C. R., Dopita, M. A., Sadler, E. M., \& Binette, L. 1986, AJ, 91, 1062

Press, W. H., Flannery, B. P., Teukolsky, S. A., \& Vetterling, W. T. 1986, Numerical Recipes (Cambridge: Cambridge Univ. Press)

Rice, W., Lonsdale, C. J., Soifer, B. T., Neugebauer, G., Kopan, E. L., Lloyd, L. A., de Jong, T., \& Habing, H. J. 1988, ApJS, 68, 91 
Rix, H.-W., \& White, S. D. M. 1992, MNRAS, 254, 389

Roberts, M. S., Hogg, D. E., Bregman, J. N., Forman, W. R., \& Jones, C. 1991, ApJS, 75, 751

Rodríguez-Espinosa, J. M., Rudy, R. J., \& Jones, B. 1987, ApJ, 312, 555

Rose, J. A., \& Searle, L. 1982, ApJ, 253, 556

Rubin, V. C., Ford, W. K., Jr., \& Thonnard, N. 1978, ApJ, 225, L107

Rush, B., Malkan, M. A., \& Spinoglio, L. 1993, ApJS, 89, 1

Sandage, A. R., \& Brucato, R. 1979, AJ, 84, 472

Sandage, A. R., \& Tammann, G. A. 1981, A Revised Shapley-Ames Catalog of Bright Galaxies (Washington, DC: Carnegie Institute of Washington) (RSA)

Sanders, D. B., Egami, E., Lipari, S., Mirabel, I. F., \& Soifer, B. T. 1995, AJ, 110, 1993

Sellwood, J. A., \& Wilkinson, A. 1993, Rep. Prog. Phys., 56, 173

Shaw, R. A., \& Dufour, R. J. 1995, PASP, 107, 896

Shields, J. C. 1992, ApJ, 399, L27

Shlosman, I. 1994, ed., Mass Transfer Induced Activity in Galaxies, (Cambridge: Cambridge Univ. Press)

Shuder, J. M. 1980, ApJ, 240, 32

Shuder, J. M., \& Osterbrock, D. E. 1981, ApJ, 250, 55

Simien, F., \& de Vaucouleurs, G. 1986, ApJ, 302, 564

Soifer, B. T., Boehmer, L., Neugebauer, G., \& Sanders, D. B. 1989, AJ, 98, 766

Spinrad, H., \& Taylor, B. J. 1971, ApJS, 22, 445

Stauffer, J. R. 1982a, ApJS, 50, 517

Stauffer, J. R. 1982b, ApJ, 262, 66

Storchi-Bergmann, T. 1991, MNRAS, 249, 404

Storchi-Bergmann, T., Bica, E., \& Pastoriza, M. G. 1990, MNRAS, 245, 749

Storchi-Bergmann, T., \& Pastoriza, M. G. 1989, ApJ, 347, 195

Storchi-Bergmann, T., \& Pastoriza, M. G. 1990, PASP, 102, 1359

Surace, J. A., Mazzarella, J., Soifer, B. T., \& Wehrle, A. E. 1993, AJ, 105, 864

Terlevich, R., Melnick, J., \& Moles, M. 1987, in Observational Evidence of Activity in Galaxies, ed. E. Ye. Khachikian, K. J. Fricke, \& J. Melnick (Dordrecht: Reidel), 499

Tully, R. B. 1987, ApJ, 321, 280

Tully, R. B. 1988, Nearby Galaxies Catalog (Cambridge: Cambridge Univ. Press)

Tully, R. B., \& Shaya, E. J. 1984, ApJ, 281, 31

van Driel, W., de Graauw, Th., de Jong, T., \& Wesselius, P. R. 1993, A\&AS, 101, 207

Veilleux, S., Kim, D.-C., Sanders, D. B., Mazzarella, J. M., \& Soifer, B. T. 1995, ApJS, 98, 171

Veilleux, S., \& Osterbrock, D. E. 1987, ApJS, 63, 295

Véron, P., \& Véron-Cetty, M.-P. 1986, A\&A, 161, 145 
Véron-Cetty, M.-P., \& Véron, P. 1986, A\&AS, 66, 335

Weedman, D. W. 1970, ApJ, 159, 405

Weedman, D. W. 1977, ARA\&A, 15, 69

Whittle, M. 1985, MNRAS, 213, 1

Whittle, M. 1992a, ApJS, 79, 49

Whittle, M. 1992b, ApJ, 387, 109

Whittle, M. 1992c, ApJ, 387, 121

Worthey, G. 1992, Ph.D. thesis, Univ. of California, Santa Cruz 


\section{Figure Captions}

Fig. 1. - Sample absorption-line spectra used as templates for starlight subtraction. The two discontinuous wavelength regions represent the blue and red spectra of our observation setup. Arbitrary constants were added to the scaled spectra for clarity

Fig. 2. - Illustration of the method of starlight subtraction. In each panel, the top plot shows the observed spectrum, the middle plot shows the best-fitting "template" model (offset by

a constant) used to match the stellar component, and the bottom plot represents the difference between the object spectrum and the model spectrum. In the case of the H II nucleus of NGC 3596 (a), the model was constructed from NGC 205 and NGC 4339, while for the Seyfert 2 nucleus of NGC $7743(b)$, the model was derived from a linear combination of NGC 205, NGC 628, and NGC 4339.

Fig. 3. - Additional examples of starlight subtraction. (a) NGC 1052. (b) NGC 2768.

Fig. 4. - Additional examples of starlight subtraction. (a) NGC 3389. (b) NGC 4419.

Fig. 5. - Flux of the narrow $\mathrm{H} \alpha$ emission line plotted against the galaxy distance. There is no obvious correlation between the two quantities. Only data of quality "b" or better are shown.

Fig. 6. - Distributions of FWHM for [N II] $\lambda 6583$ as a function of the seeing conditions (FWHM of the seeing disk in seconds of arc). Only FWHM([N II $]$ ) values of quality "b" or better are shown. The top panel combines measurements for all emission-line objects, while the bottom four panels plot each of the spectral classes individually.

Fig. 7. - (a) Diagnostic diagram plotting log [O III $] \lambda 5007 / \mathrm{H} \beta$ versus $\log [\mathrm{N}$ II $] \lambda 6583 / \mathrm{H} \alpha$ for H II nuclei (crosses), Seyfert nuclei (squares), LINERs (solid circles), and transition objects (open circles). (b) Diagnostic diagram plotting $\log [\mathrm{O}$ III $] \lambda 5007 / \mathrm{H} \beta$ versus $\log [\mathrm{S} \mathrm{II}] \lambda \lambda 6716$, $6731 / \mathrm{H} \alpha$. Symbols same as in $(a)$. (c) Diagnostic diagram plotting log [O III] $\lambda 5007 / \mathrm{H} \beta$ versus $\log [\mathrm{O}$ I $] \lambda 6300 / \mathrm{H} \alpha$. Symbols same as in $(a)$.

Fig. 8. - Sample starlight-subtracted spectra of H II nuclei.

Fig. 9. - Sample starlight-subtracted spectra of LINERs.

Fig. 10. - Sample starlight-subtracted spectra of transition objects.

Fig. 11. - Sample starlight-subtracted spectra of Seyfert 2 nuclei.

Fig. 12. - Sample starlight-subtracted spectra of Seyfert 1 nuclei.

Fig. 13. - Distribution of [O I] $\lambda 6300 /[\mathrm{O}$ III] $\lambda 5007$ for the 64 LINERs in the Palomar survey with reliable measurements of both lines. Line ratios having quality rating "c" and all upper limits were excluded.

Fig. 14. - Correlations among the low-ionization optical forbidden lines. (a) Log [N II] $\lambda 6583 / \mathrm{H} \alpha$ vs. $\log [\mathrm{O} \mathrm{I}] \lambda 6300 / \mathrm{H} \alpha .(b) \log [\mathrm{S} \mathrm{II}] \lambda 6725 / \mathrm{H} \alpha$ vs. $\log [\mathrm{O} \mathrm{I}] \lambda 6300 / \mathrm{H} \alpha([\mathrm{S} \mathrm{II}] \lambda 6725$ $\equiv[\mathrm{S} \mathrm{II}] \lambda \lambda 6716,6731)$. Data with quality "b" or better are shown as filled dots, and those with quality "c" are shown as open dots.

Fig. 15. - Distribution of [N II $] \lambda 6583 / \mathrm{H} \alpha$ as a function of galaxy distance. Only $[\mathrm{N} \mathrm{II}] / \mathrm{H} \alpha$ values of quality "b" or better are shown. The top panel combines measurements for all 
emission-line objects, while the bottom five panels plot each of the spectral classes separately, as well as the three AGN classes combined.

Fig. 16. - Definition of the stellar absorption-line indices for the $(a)$ blue and $(b)$ red spectral regions.

Fig. 17. - Distribution of morphological types for the Palomar survey. The top panel shows all 486 galaxies in the sample, the middle panel the 252 unbarred galaxies, and the bottom panel the 234 barred $(\mathrm{AB}+\mathrm{B})$ galaxies. The bottom abscissa gives the Hubble types, and the top abscissa the corresponding $\mathrm{T}$ indices, both binned slightly following the convention in Table 13.

Fig. 18. - Distribution of distances. The width of each bin corresponds to $5 \mathrm{Mpc}$.

Fig. 19. - Distribution of heliocentric radial velocities. The width of each bin corresponds to $500 \mathrm{~km} \mathrm{~s}^{-1}$.

Fig. 20. - Distribution of total apparent blue magnitudes $\left(B_{T}\right)$. The width of each bin corresponds to 0.5 mag.

Fig. 21. - Distribution of absolute blue magnitudes for the entire galaxy $\left(M_{B_{T}}^{0}\right)$, corrected for Galactic and internal extinction. The width of each bin corresponds to 0.5 mag.

Fig. 22. - Distribution of absolute blue magnitudes of the bulge component $\left[M_{B}(\mathrm{bul})\right]$. The width of each bin corresponds to 0.5 mag.

Fig. 23. - Distribution of the cosine of the galaxy inclination angles $(i)$. The width of each bin corresponds to 0.1 in $\cos i$. 


\section{NOTE CONCERNING THE TABLES}

Please note that the following tables are not included in this preprint: Tables 2, 3, 4, 8, 9, 10, 11, and 12. These tables are very large, containing nearly 40,000 data entries. The entries are being checked for accuracy and occasionally still being revised. We will not distribute these tables prior to the actual publication of the paper. We apologize for any inconvenience. 


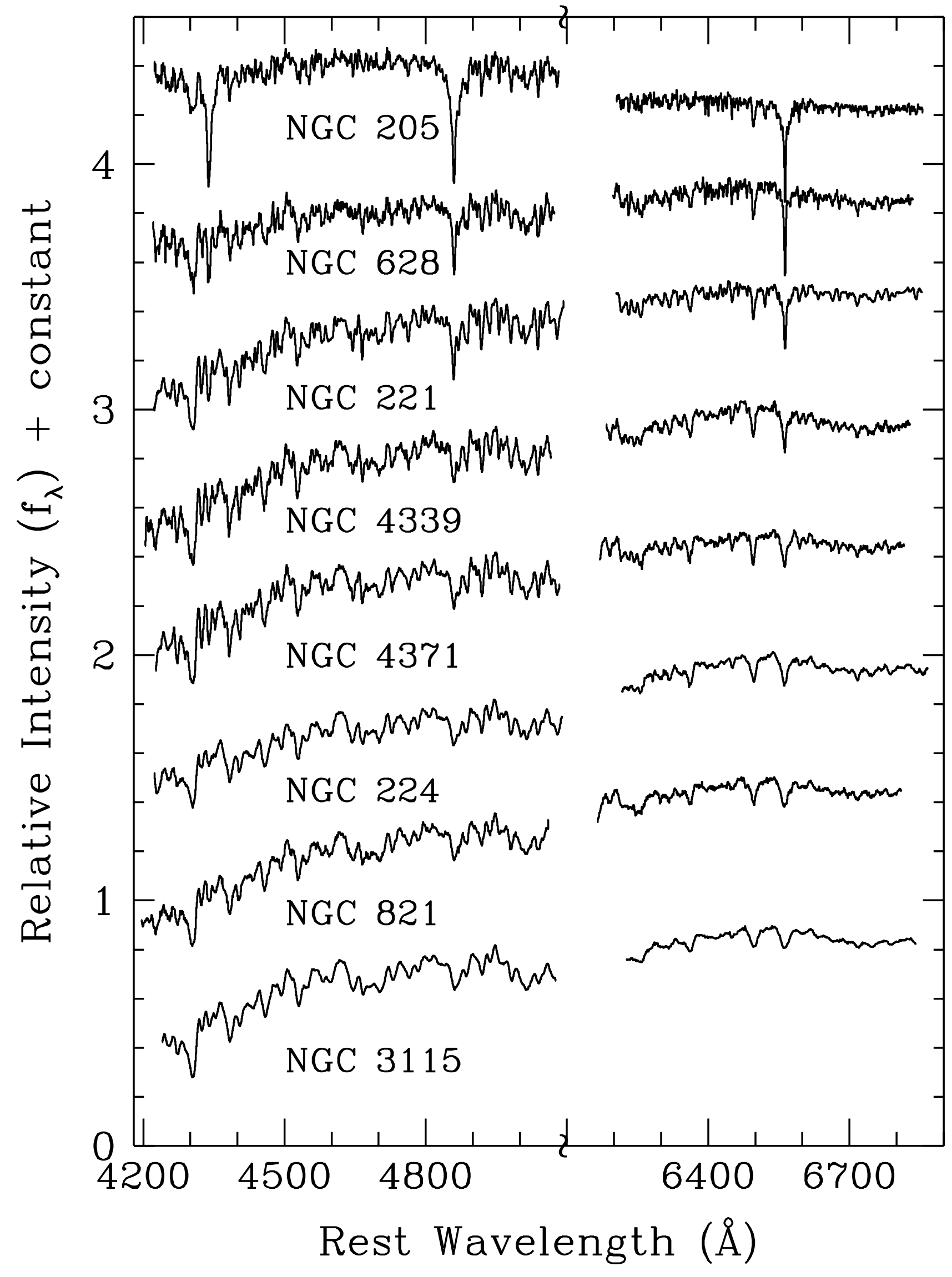




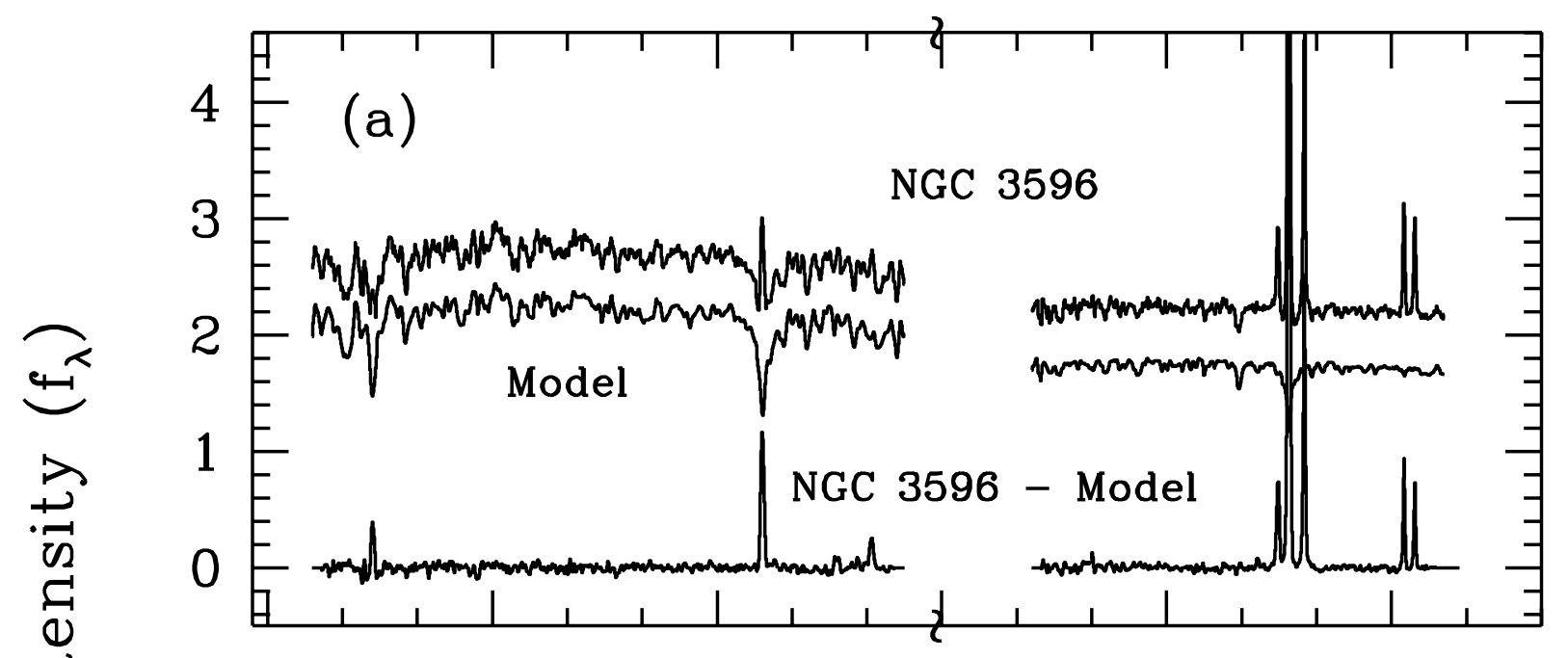

트

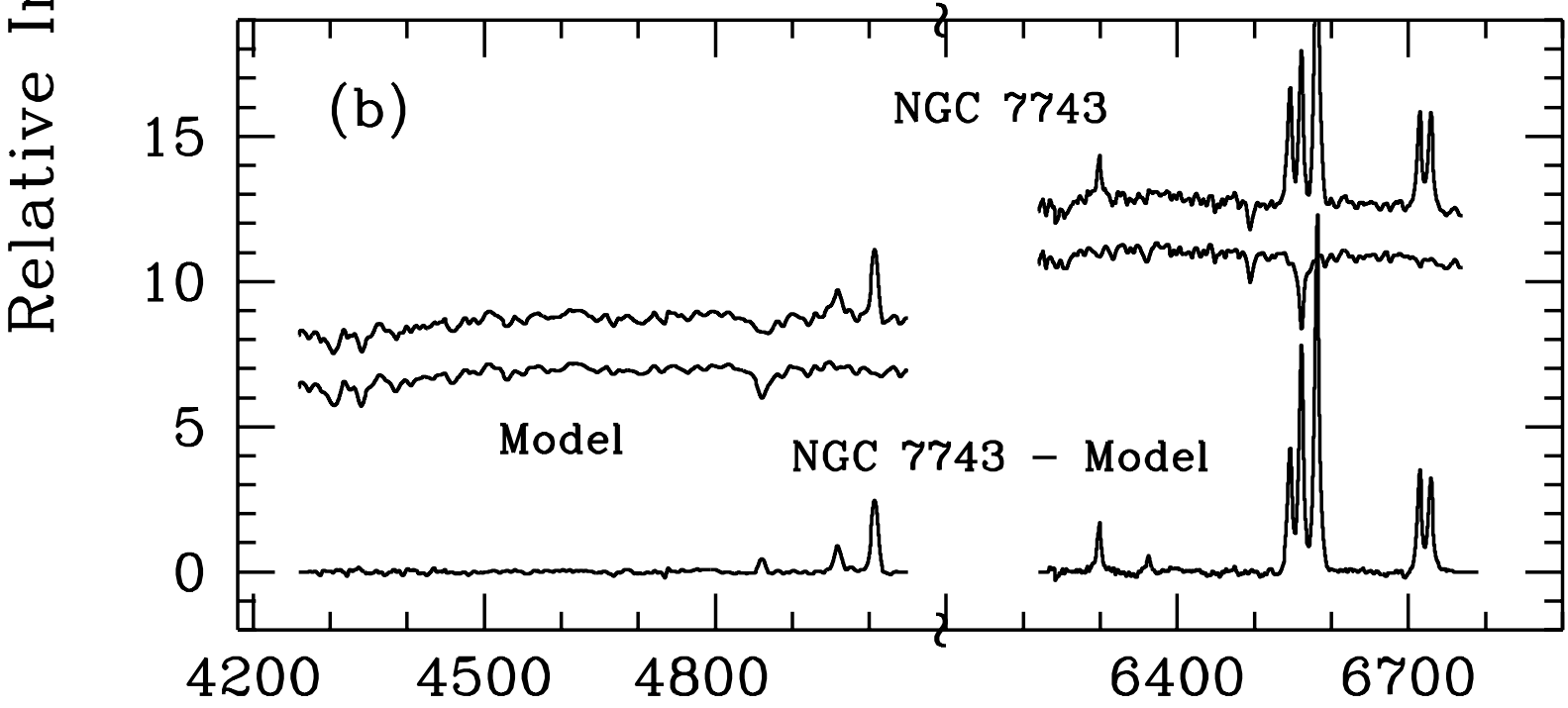

Rest Wavelength $(\AA)$ 


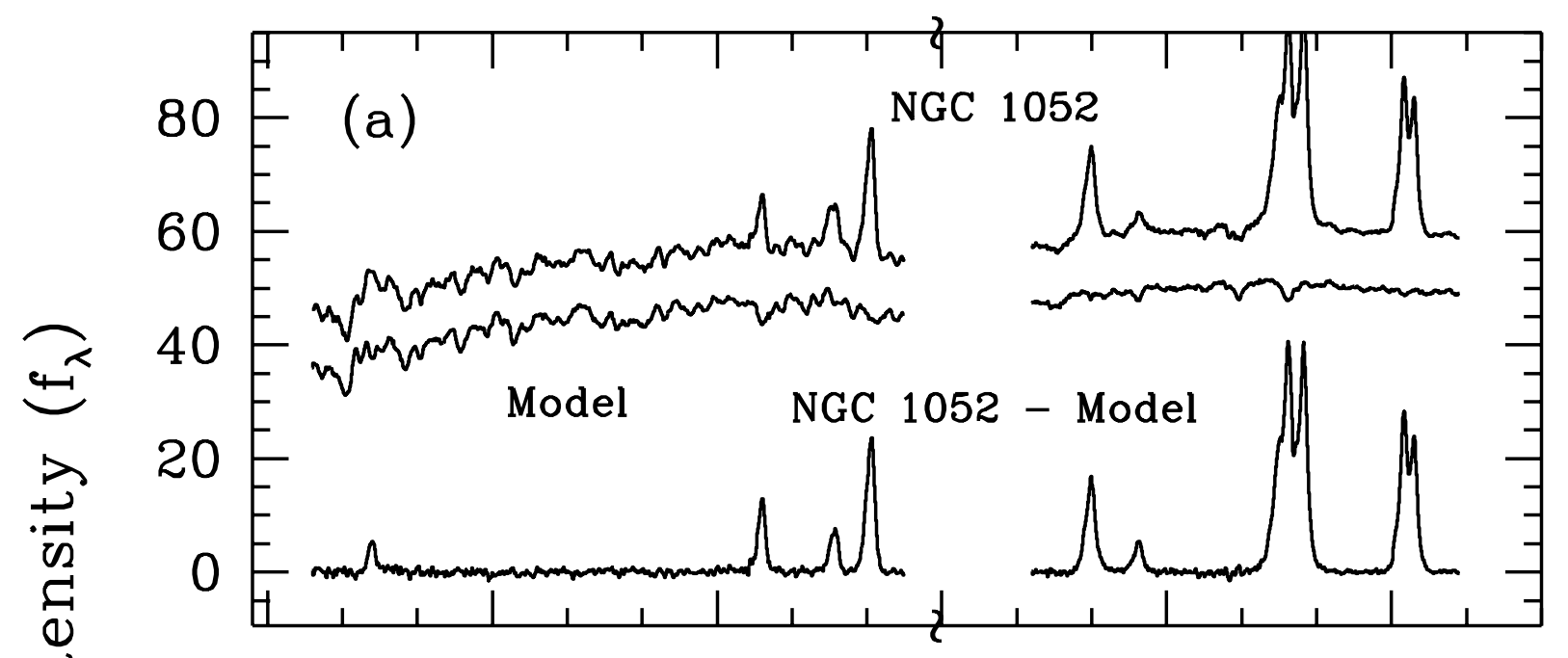

تص

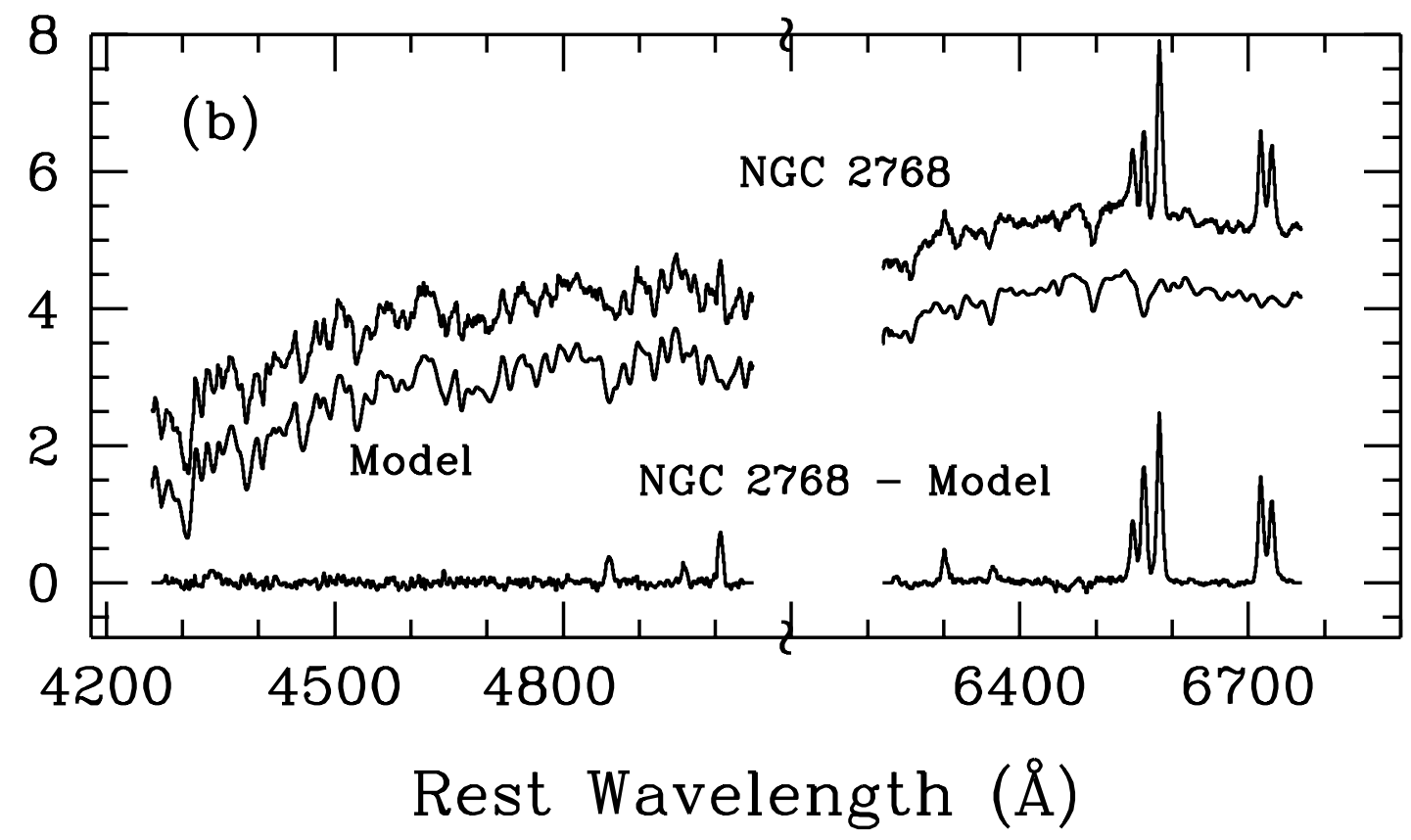




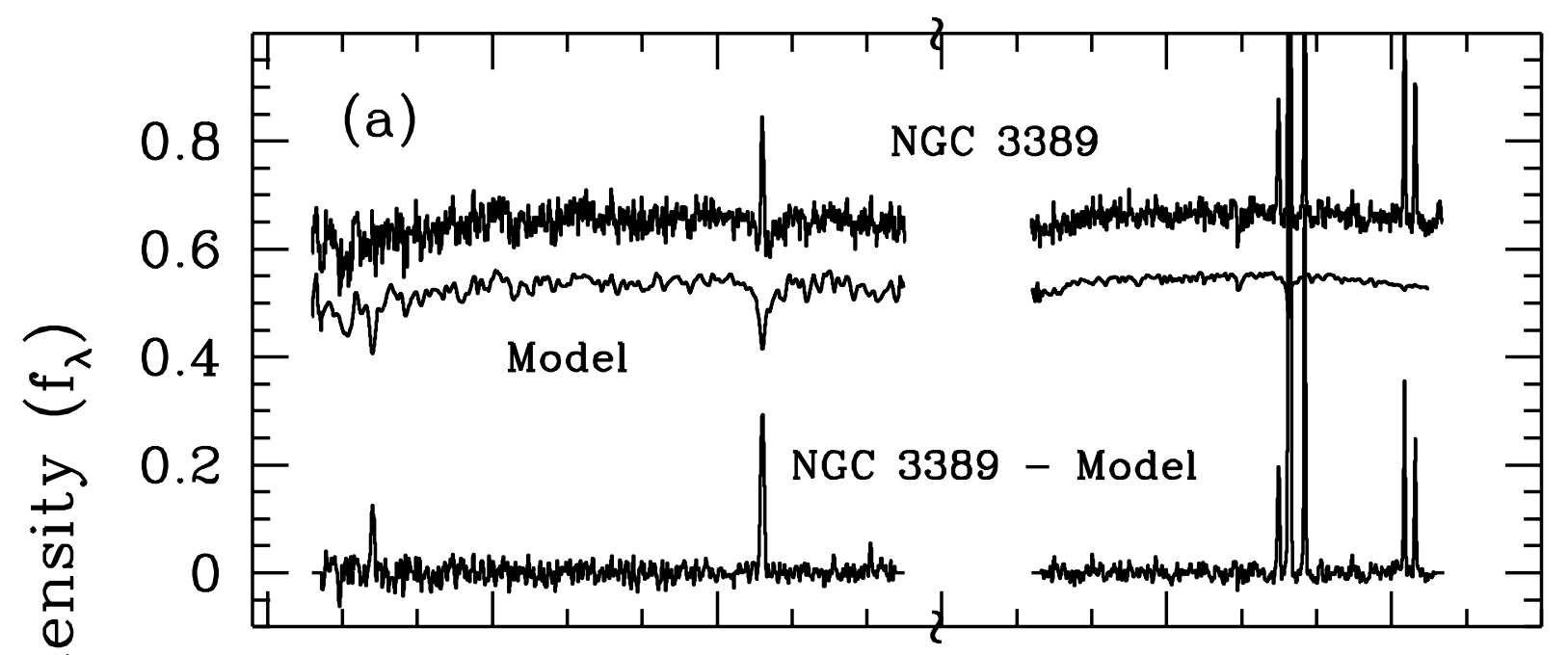

ש

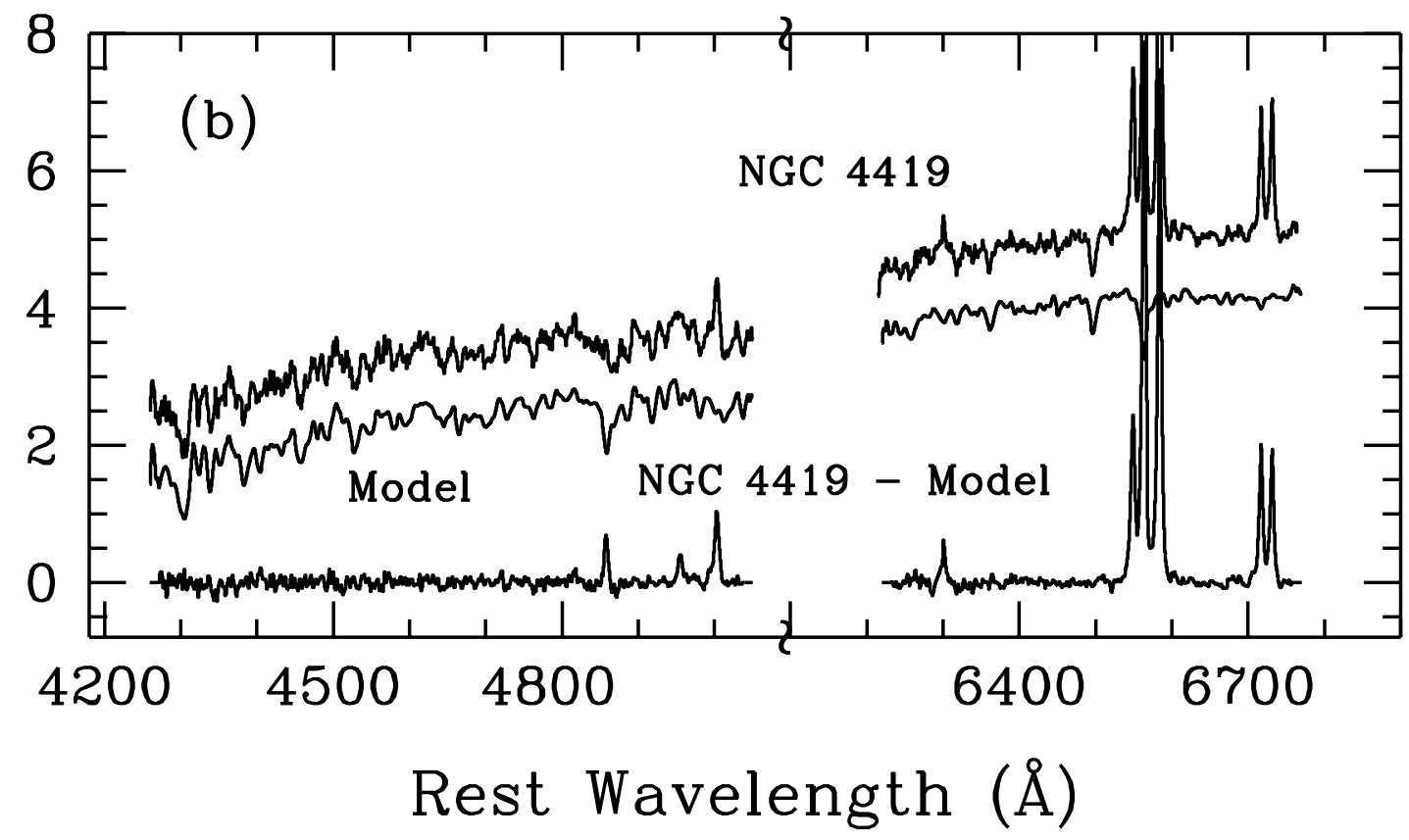




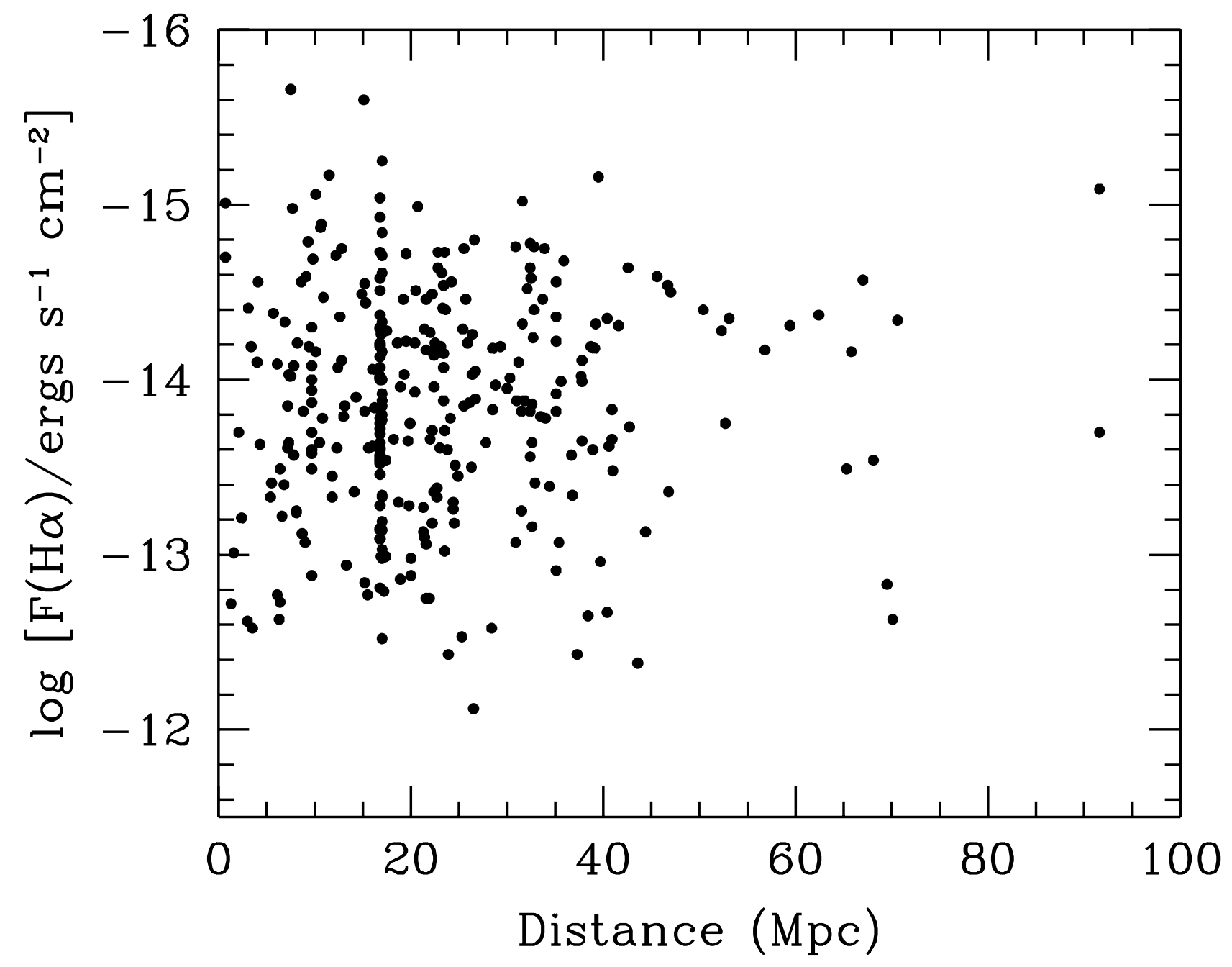


TABLE 5

CRITERIA FOR SPECTRAL CLASSIFICATION

\begin{tabular}{lcccc}
\hline \hline \multicolumn{1}{c}{ Class } & {$[\mathrm{O} \mathrm{III]} / \mathrm{H} \beta$} & {$[\mathrm{O} \mathrm{I}] / \mathrm{H} \alpha$} & {$[\mathrm{N} \mathrm{II}] / \mathrm{H} \alpha$} & {$[\mathrm{S} \mathrm{II}] / \mathrm{H} \alpha$} \\
\hline H II nuclei & Any & $<0.08$ & $<0.6$ & $<0.4$ \\
Seyfert nuclei & $\geq 3$ & $\geq 0.08$ & $\geq 0.6$ & $\geq 0.4$ \\
LINERs & $<3$ & $\geq 0.17$ & $\geq 0.6$ & $\geq 0.4$ \\
Transition nuclei & $<3$ & $\geq 0.08,<0.17$ & $\geq 0.6$ & $\geq 0.4$ \\
\hline
\end{tabular}

Note.-Line ratios are dereddened values. $[\mathrm{O}$ III $] \equiv[\mathrm{O}$ III $] \lambda 5007,[\mathrm{O} \mathrm{I}] \equiv\left[\begin{array}{ll}\mathrm{O} & \mathrm{I}\end{array}\right] \lambda 6300,[\mathrm{~N}$ II $] \equiv$ $[\mathrm{N}$ II] $\lambda 6583,[\mathrm{~S}$ II $] \equiv[\mathrm{S} \mathrm{II}] \lambda \lambda 6716,6731$, and $\mathrm{H} \alpha$ and $\mathrm{H} \beta$ pertain only to the narrow component of the lines. 


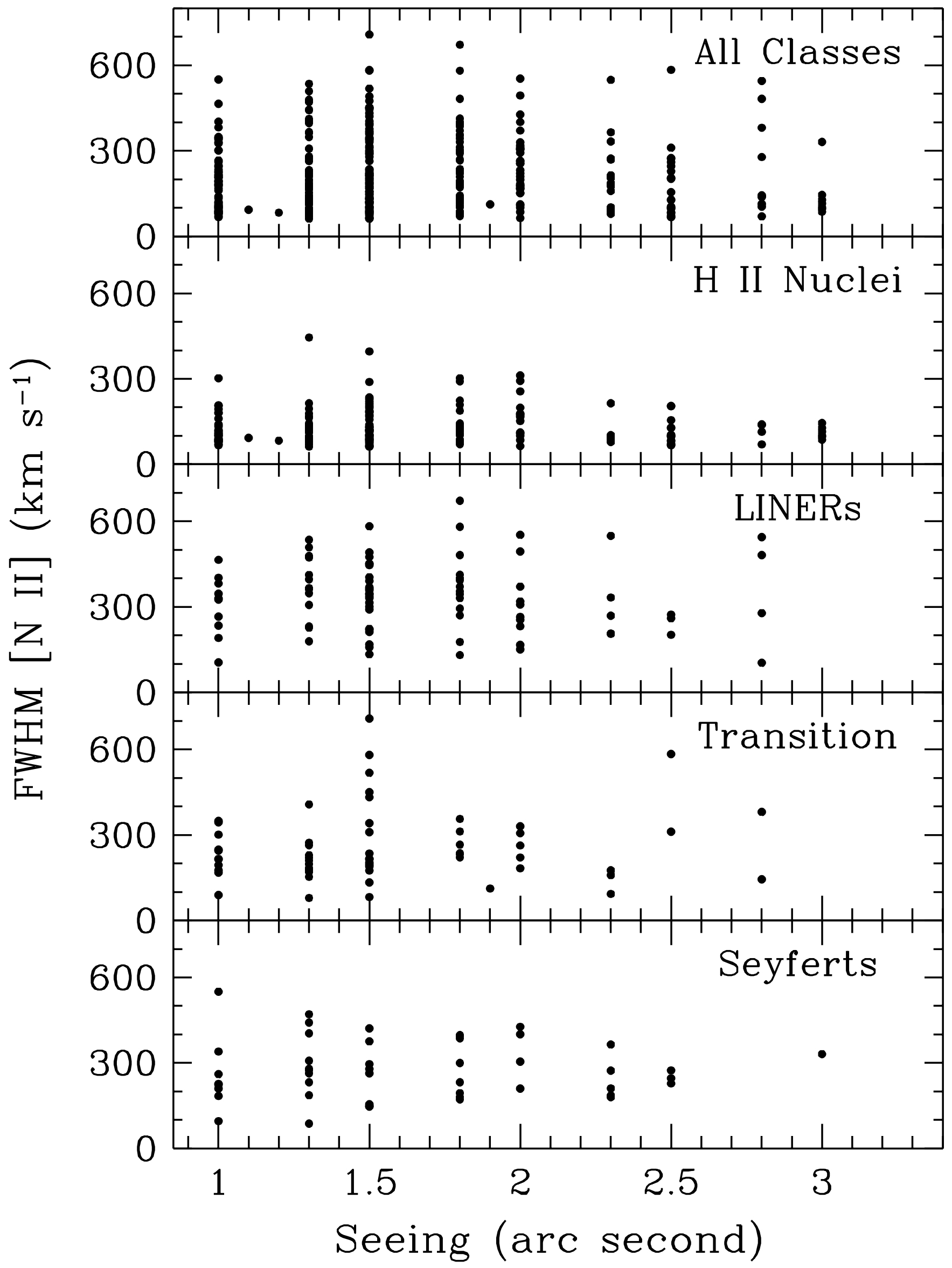


TABLE 6

DEFINITION OF CONTINUUM BANDS

\begin{tabular}{lccc}
\hline \hline Band & $\begin{array}{c}\lambda_{c} \\
(\AA)\end{array}$ & $\begin{array}{c}\text { Region 1 } \\
(\AA)\end{array}$ & $\begin{array}{c}\text { Region 2 } \\
(\AA)\end{array}$ \\
\hline$m_{44}$ & 4340 & $4262-4281$ & $4360-4375$ \\
$m_{49}$ & 4861 & $4828-4848$ & $4880-4900$ \\
$m_{66}$ & 6563 & $6518-6538$ & $6595-6620$ \\
\hline
\end{tabular}




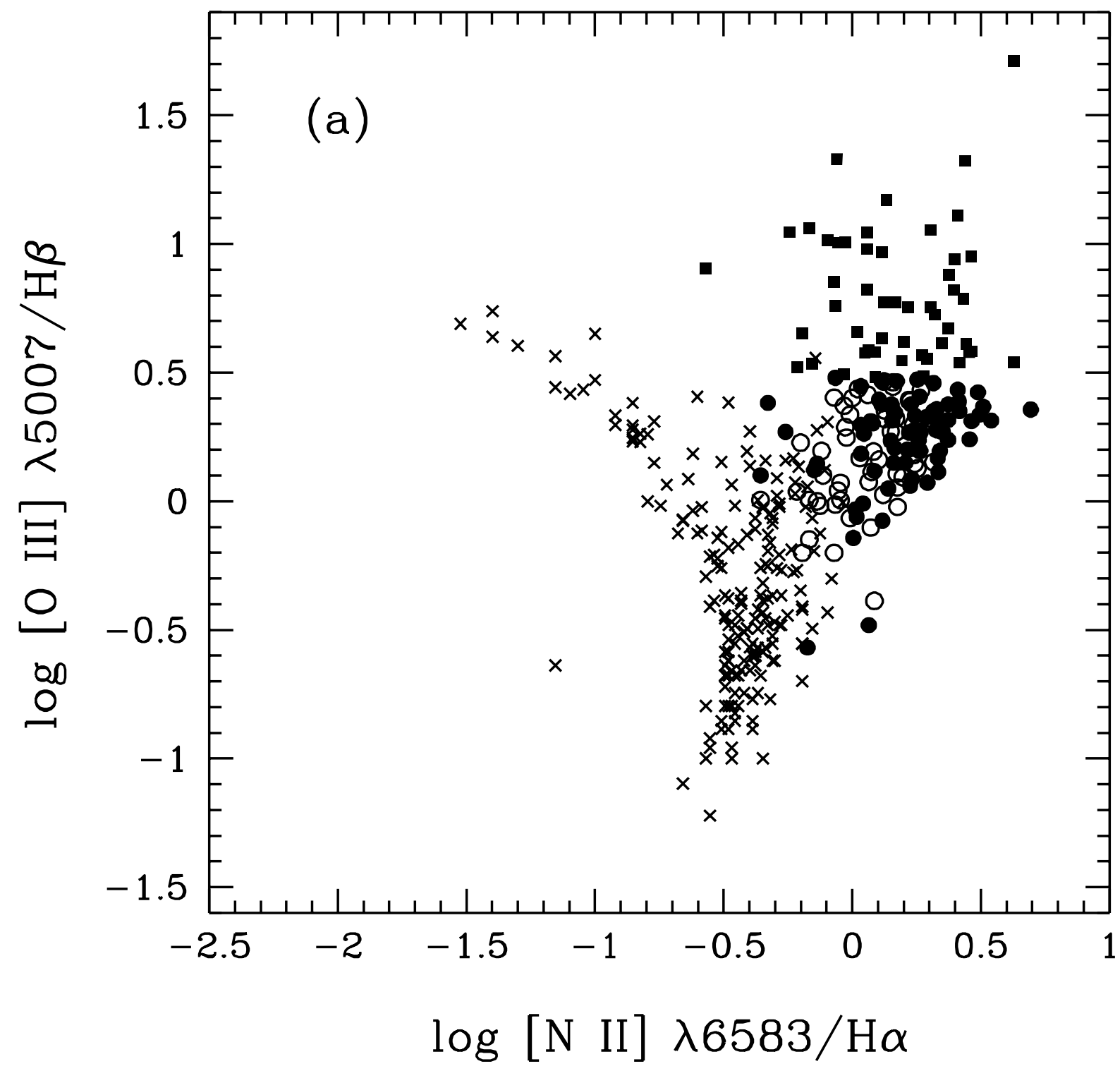




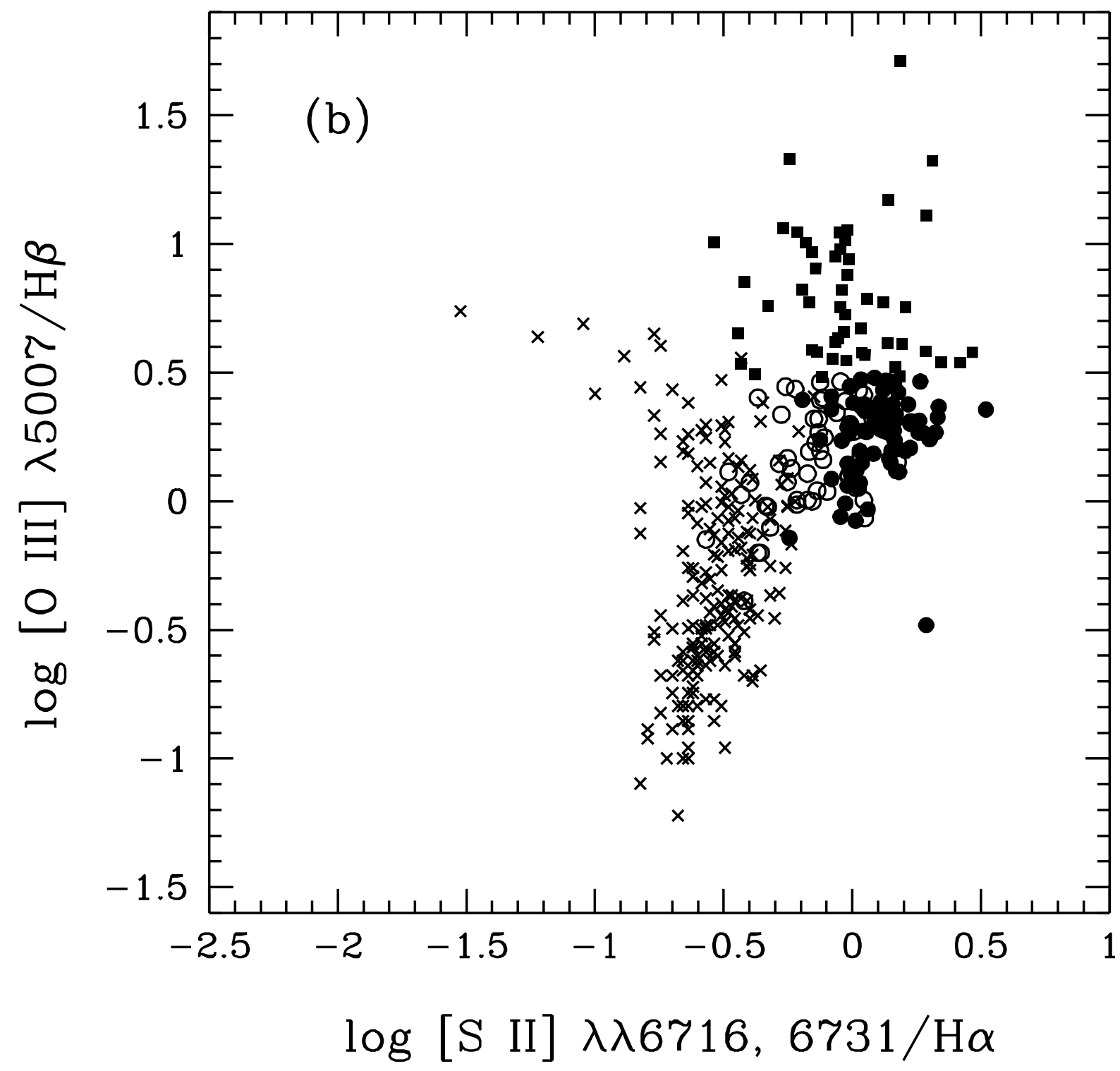




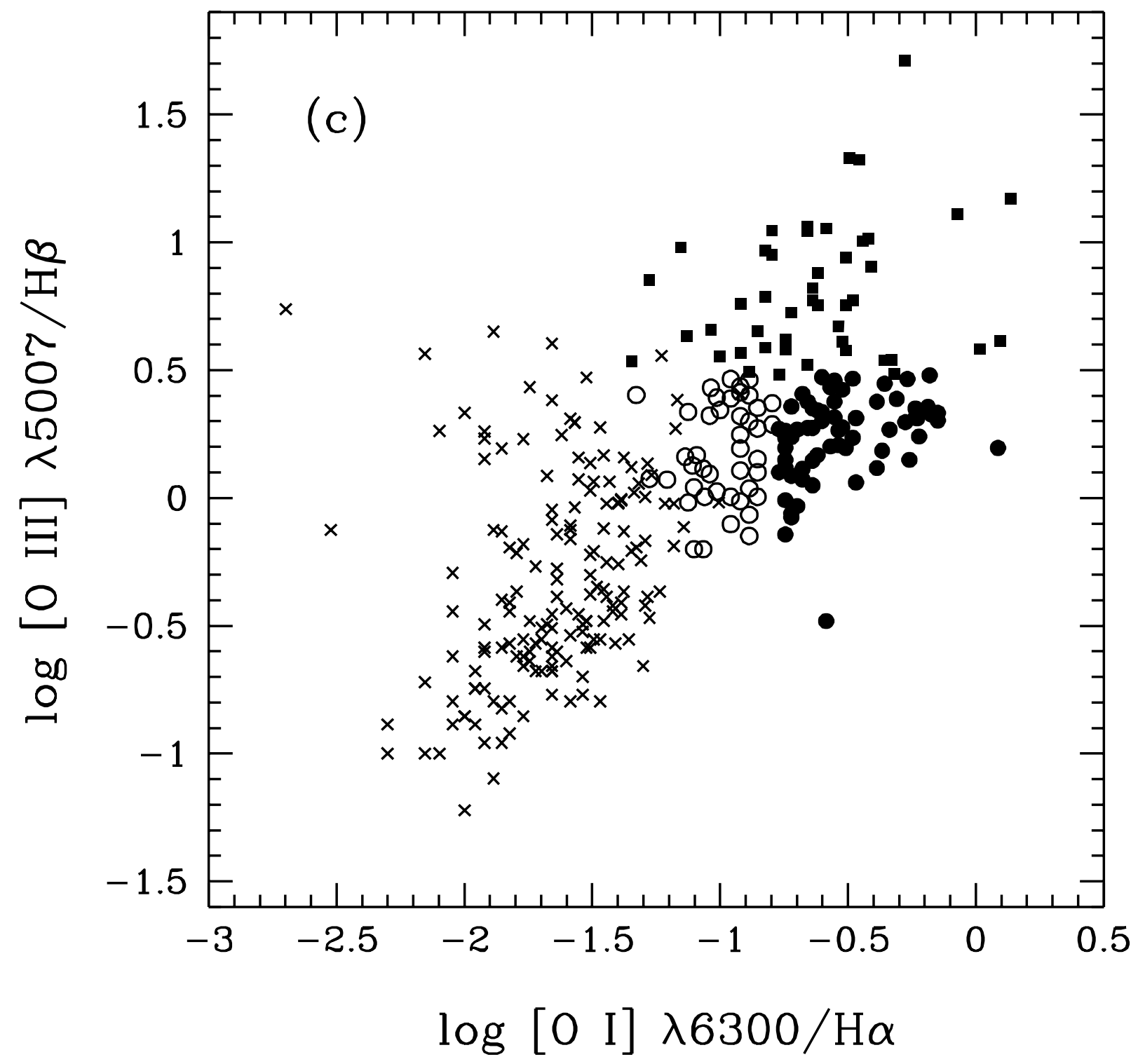


TABLE 7

DEFINITION OF STELLAR ABSORPTION-LINE INDICES

\begin{tabular}{lcccr}
\hline \hline \multicolumn{1}{c}{ Index } & $\begin{array}{c}\text { Continuum } 1 \\
(\AA)\end{array}$ & $\begin{array}{c}\text { Feature } \\
(\AA)\end{array}$ & $\begin{array}{c}\text { Continuum } 2 \\
(\AA)\end{array}$ & Ref. \\
\hline W(Gband) & $4267.6-4282.6$ & $4282.6-4317.6$ & $4320.1-4336.4$ & 1 \\
W(H $\gamma)$ & $4316.0-4321.0$ & $4333.0-4348.0$ & $4360.0-4370.0$ & 2 \\
W(Fe4383) & $4360.4-4370.4$ & $4370.4-4421.6$ & $4444.1-4456.6$ & 1 \\
W(Ca4455) & $4444.0-4448.5$ & $4453.4-4475.9$ & $4478.0-4486.0$ & 1 \\
W(Fe4531) & $4504.5-4515.5$ & $4515.5-4560.5$ & $4561.8-4580.5$ & 1 \\
W(Fe4668) & $4612.8-4631.5$ & $4635.3-4721.5$ & $4744.0-4757.8$ & 1 \\
W(H $\beta)$ & $4828.9-4848.9$ & $4848.9-4877.6$ & $4877.6-4892.6$ & 1 \\
W(Ca+Fe) & $6462.0-6475.0$ & $6475.0-6515.0$ & $6515.0-6530.0$ & 3 \\
W(H $\alpha)$ & $6515.0-6530.0$ & $6535.0-6585.0$ & $6585.0-6600.0$ & 3 \\
\hline
\end{tabular}

Refarences.-(1) Worthey 1992; (2) Held \& Mould 1994; (3) this paper. 


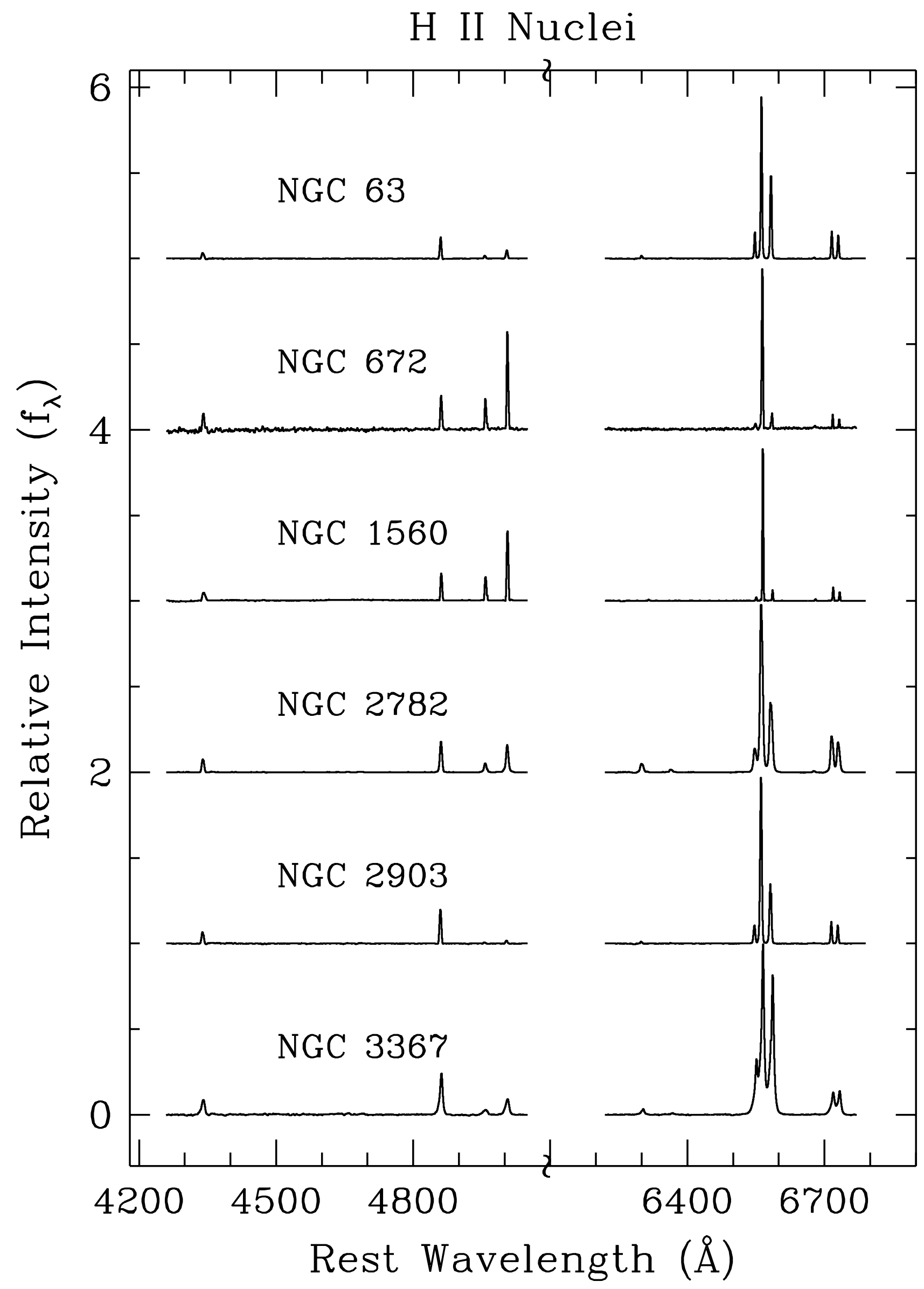


LINERs

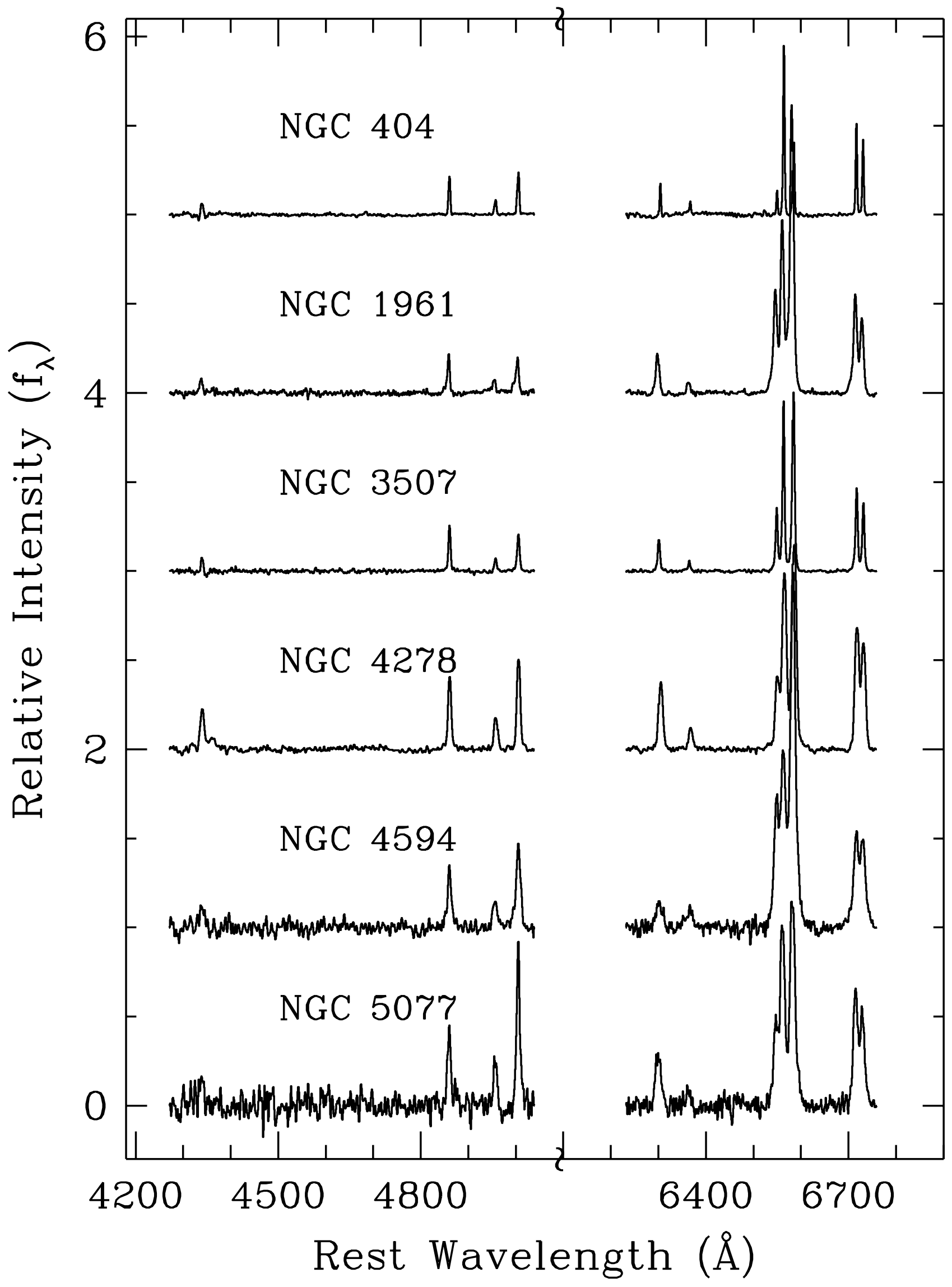




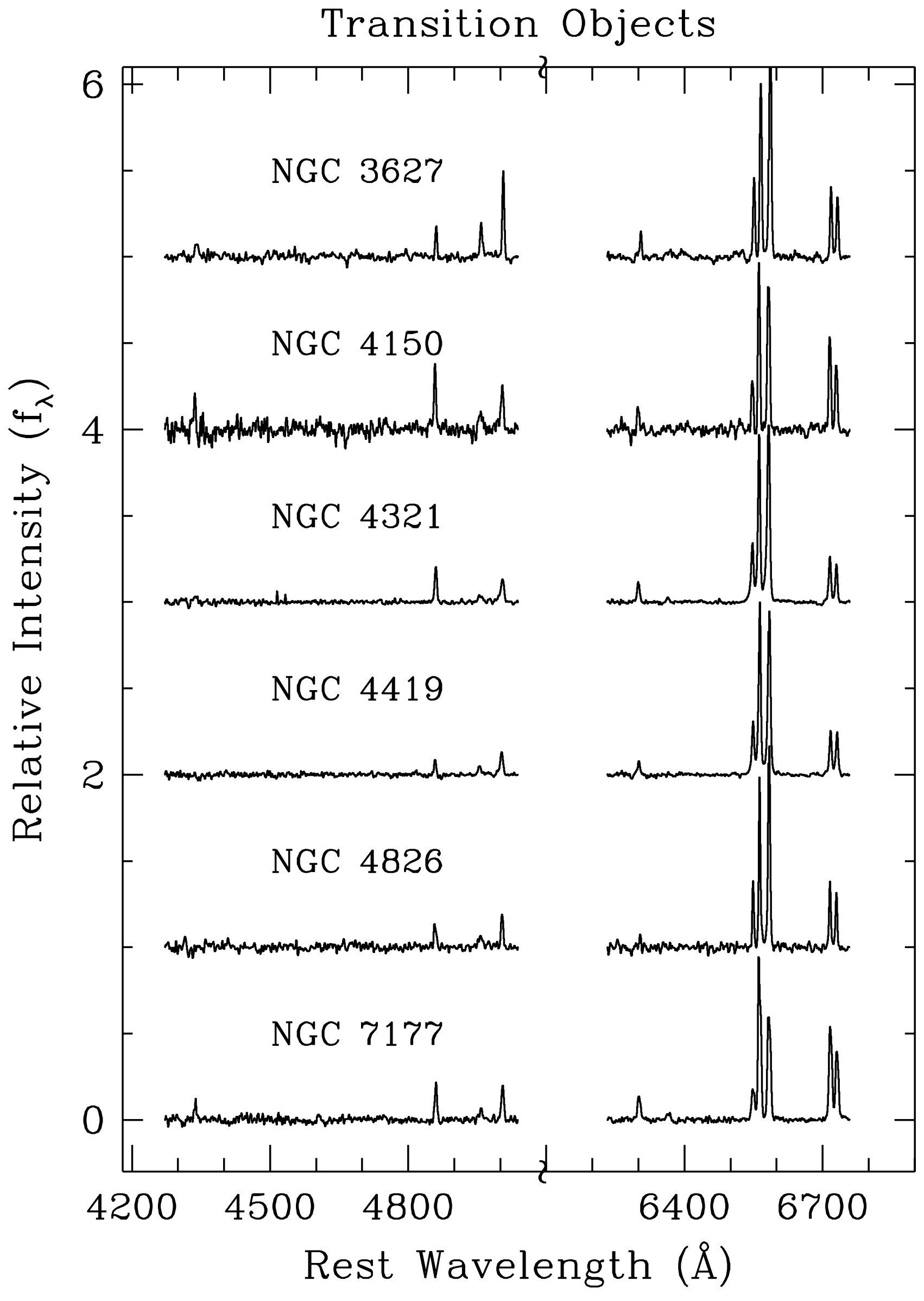


Seyfert 2 Nuclei

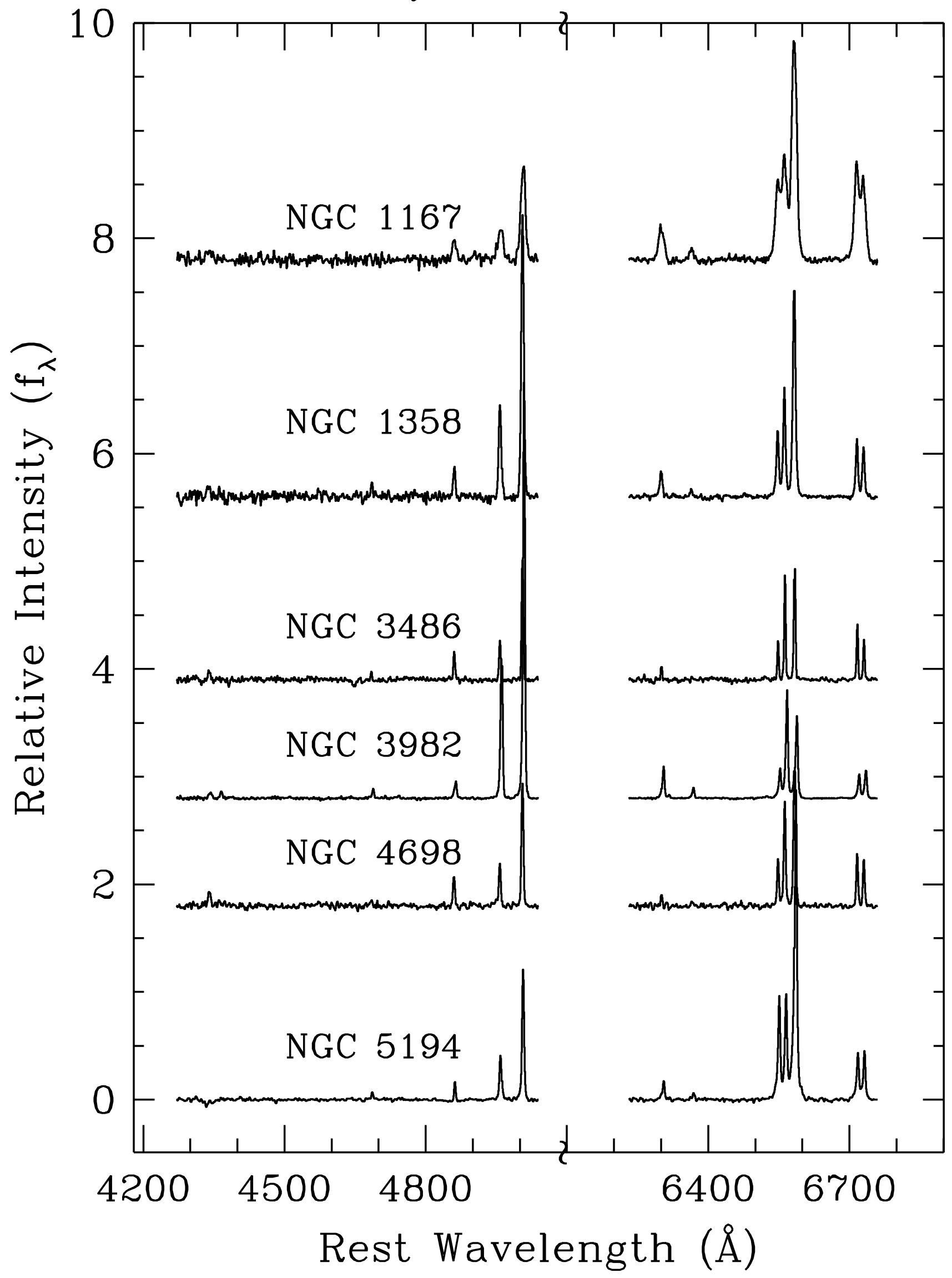


Seyfert 1 Nuclei

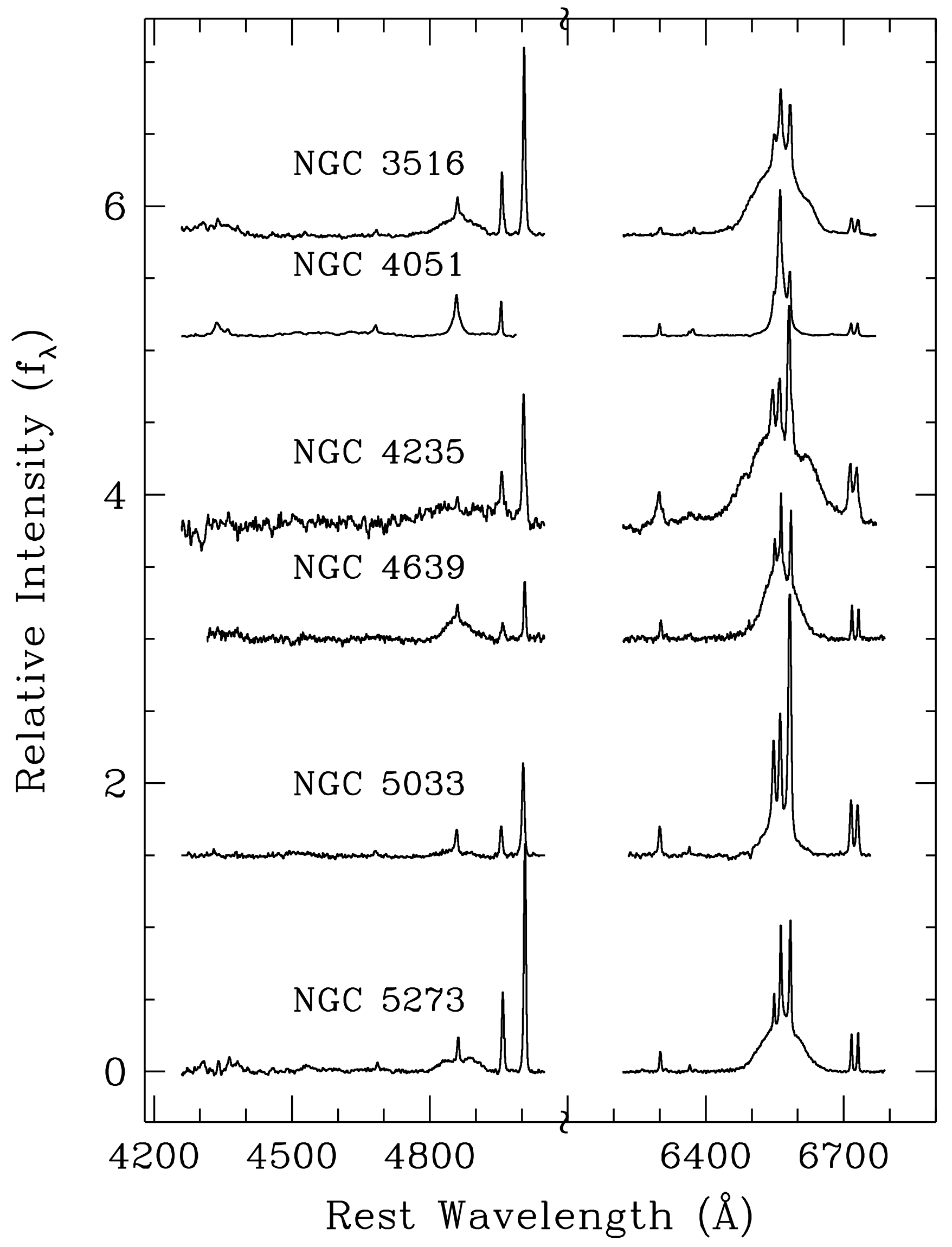




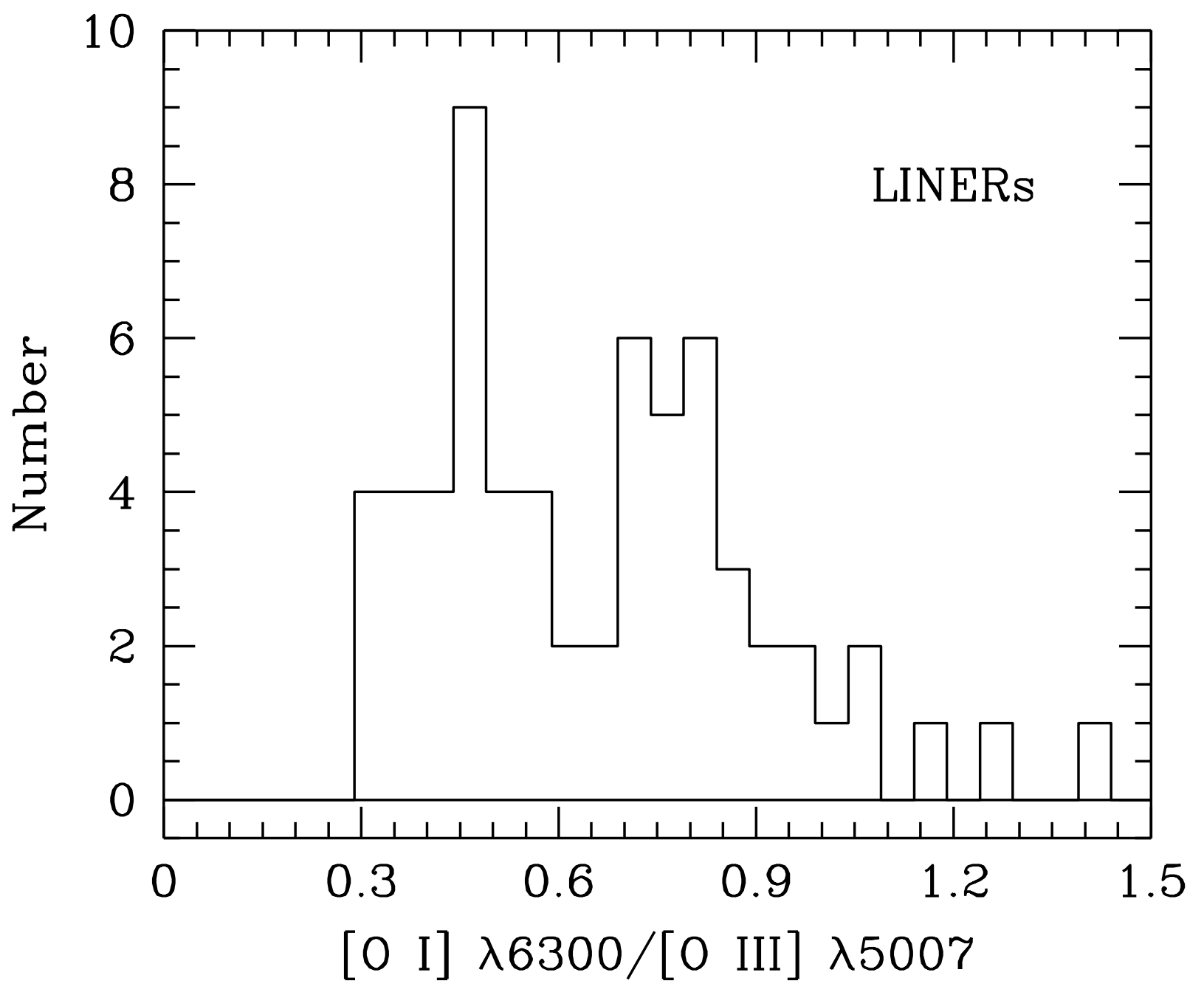



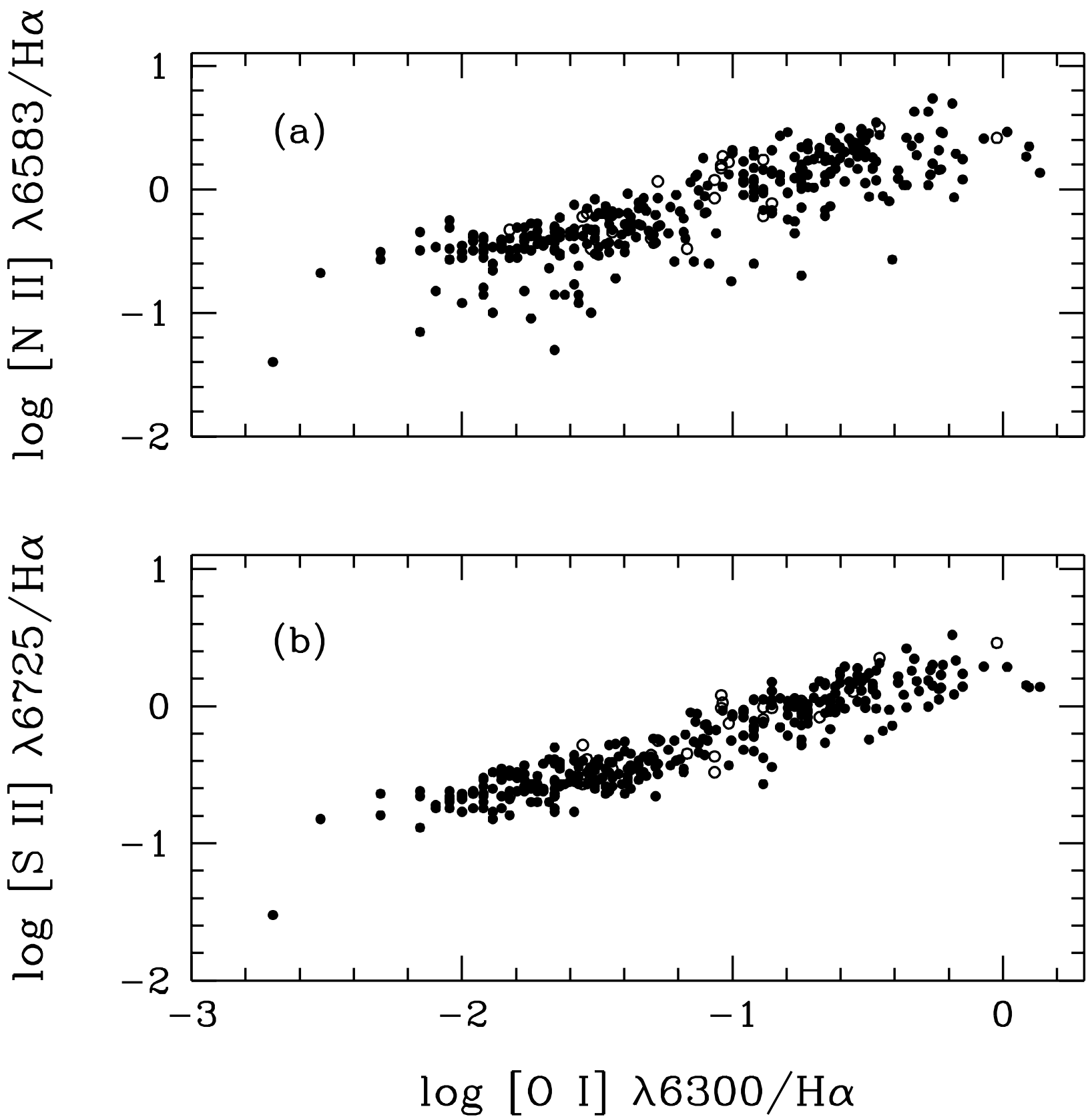


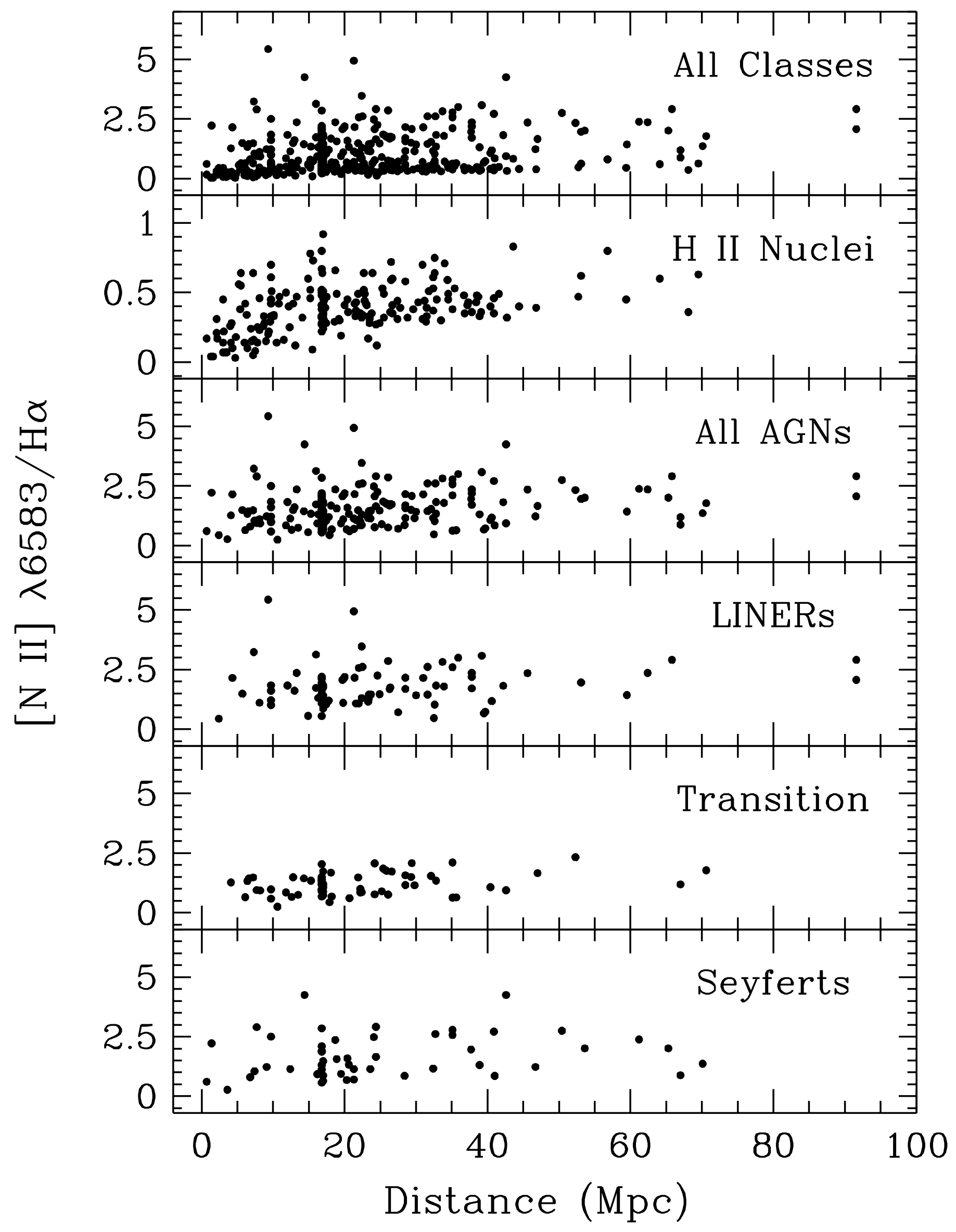


(a) Blue Stellar Indices

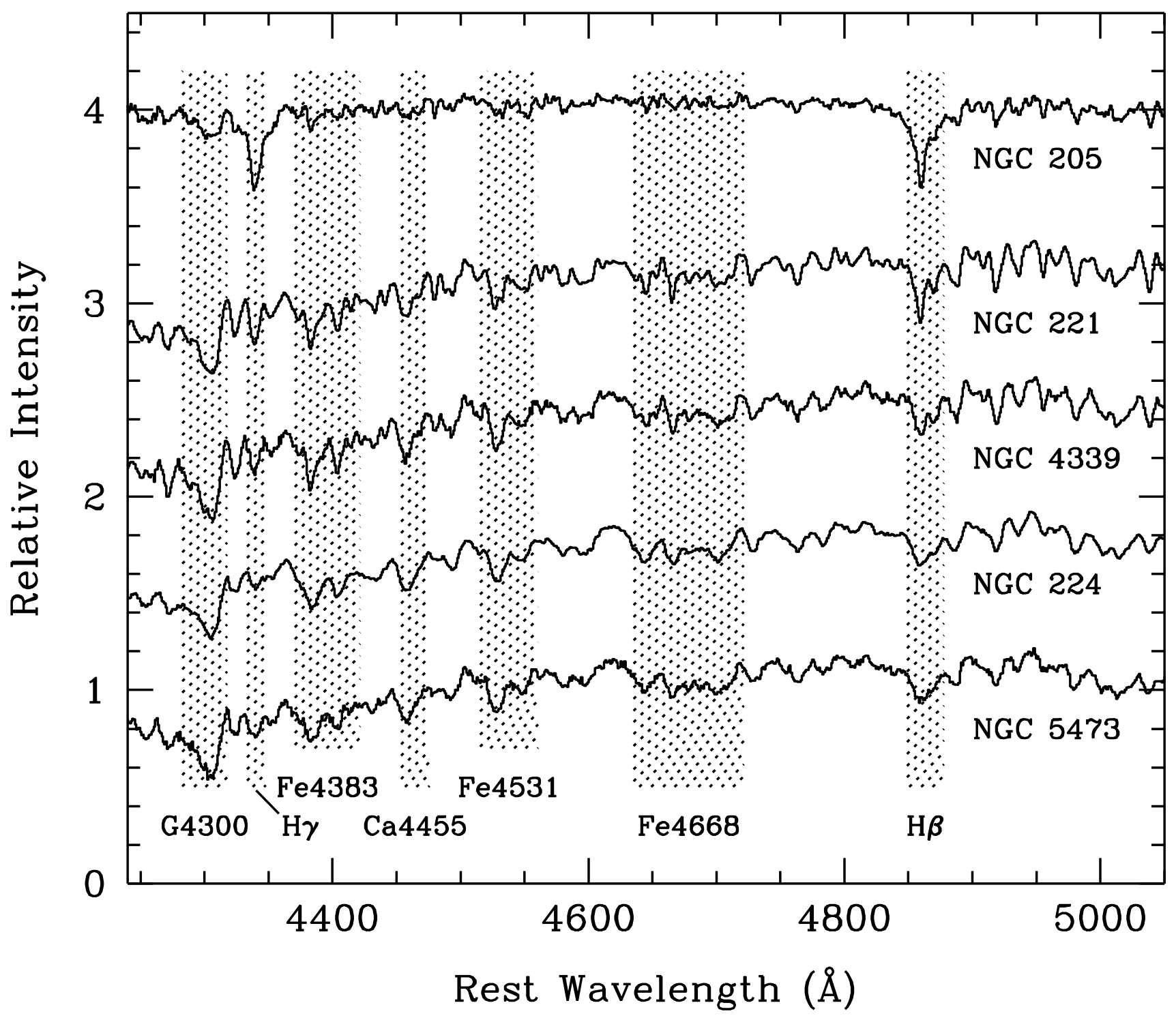


(b) Red Stellar Indices

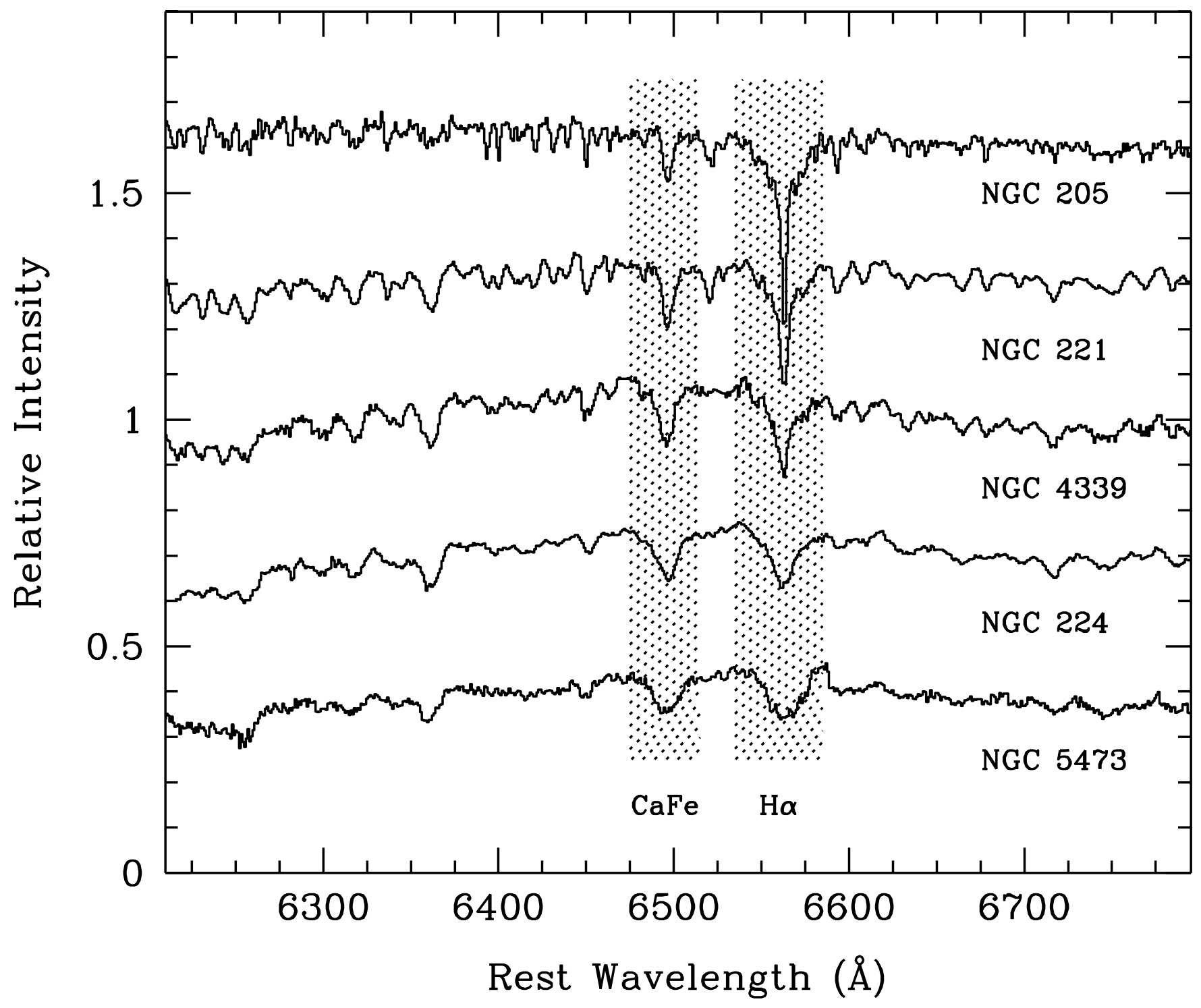




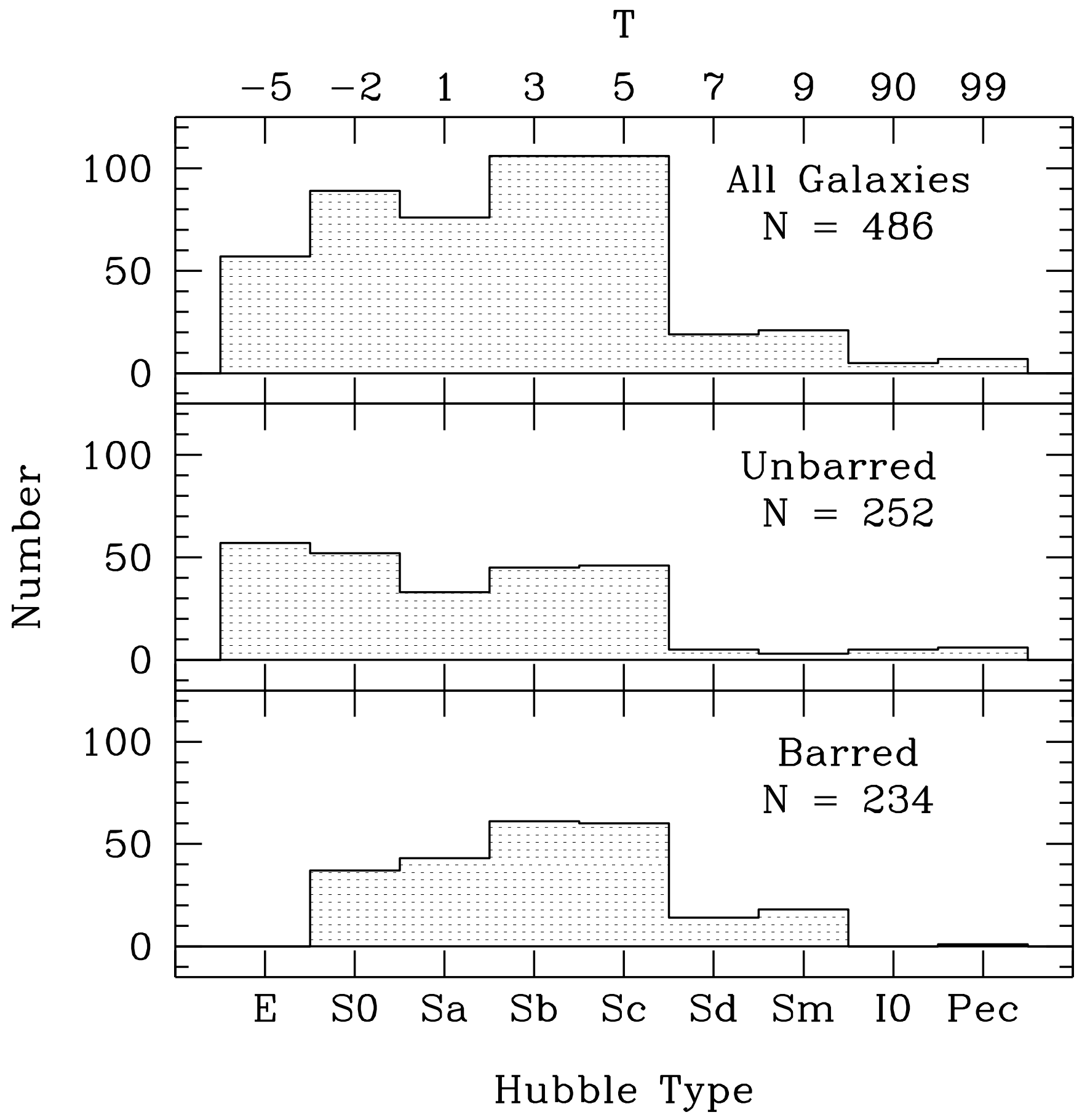




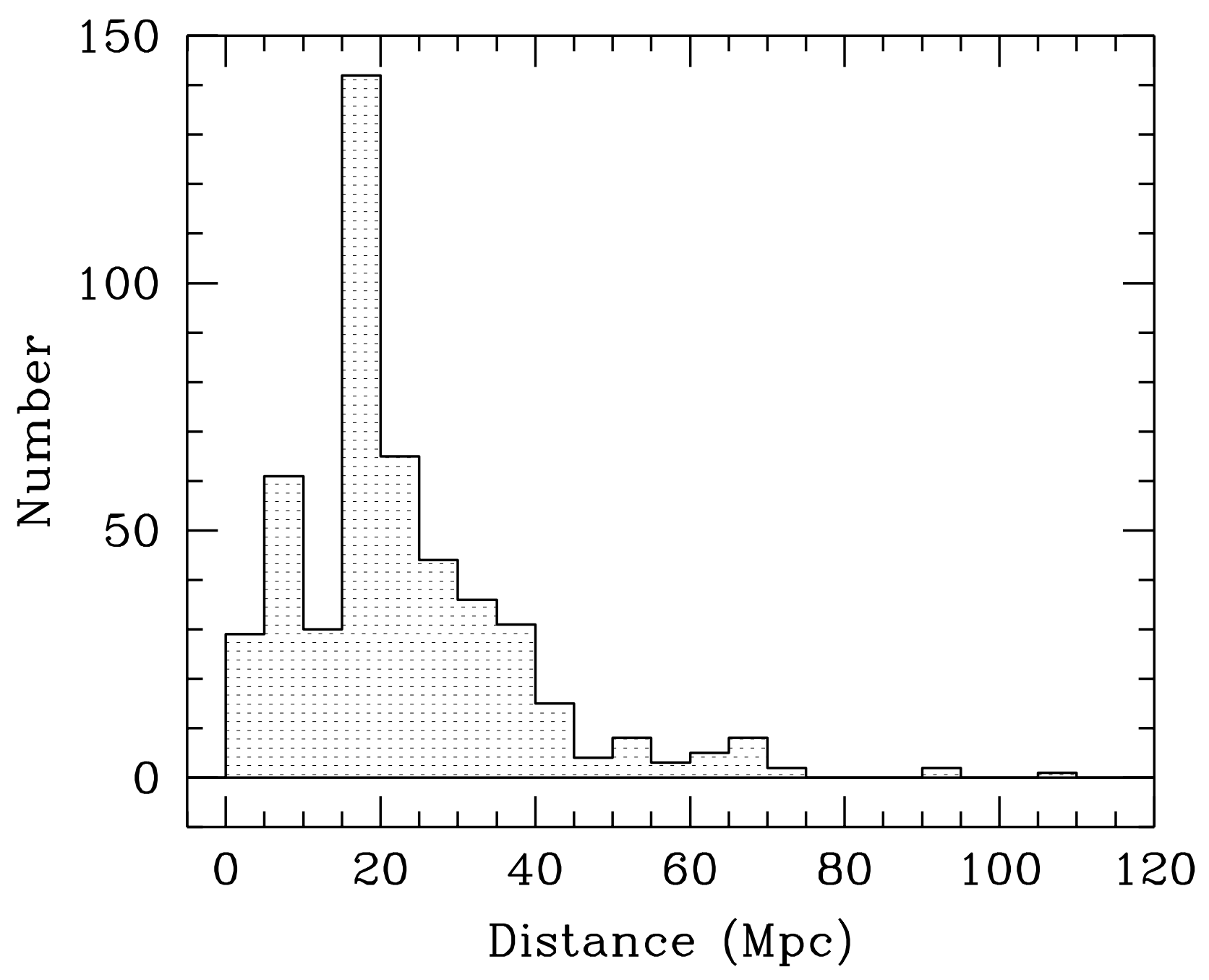




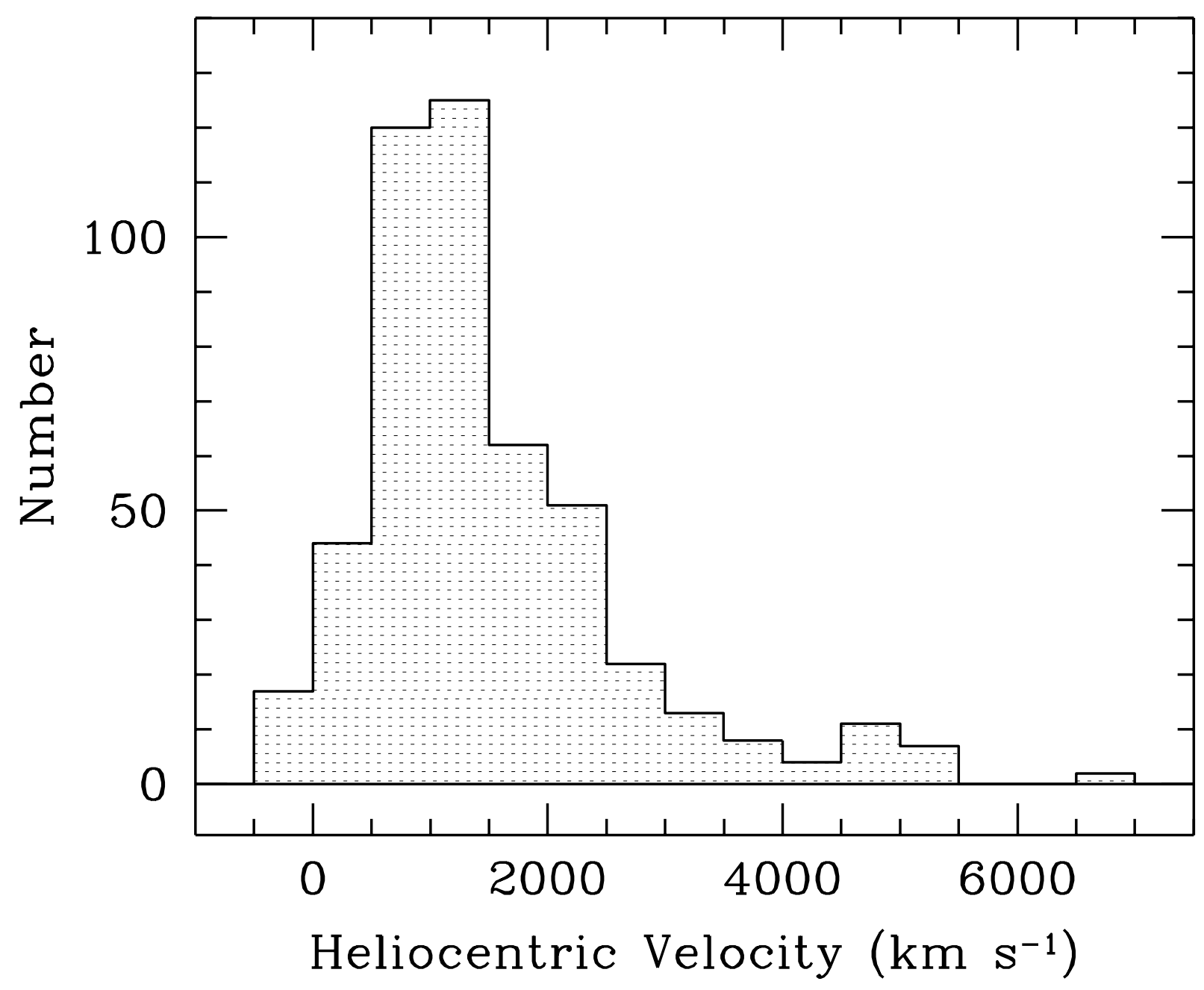




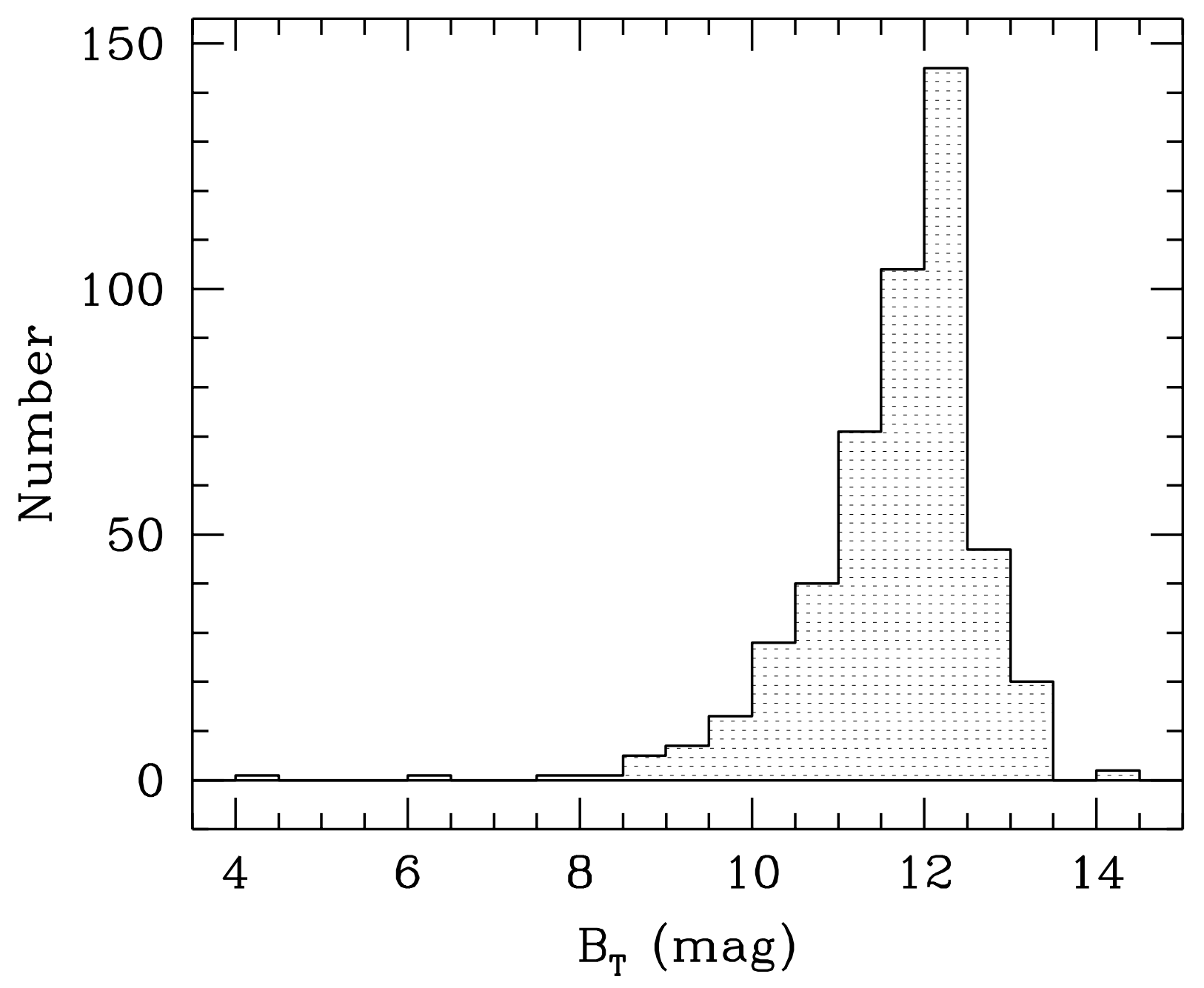




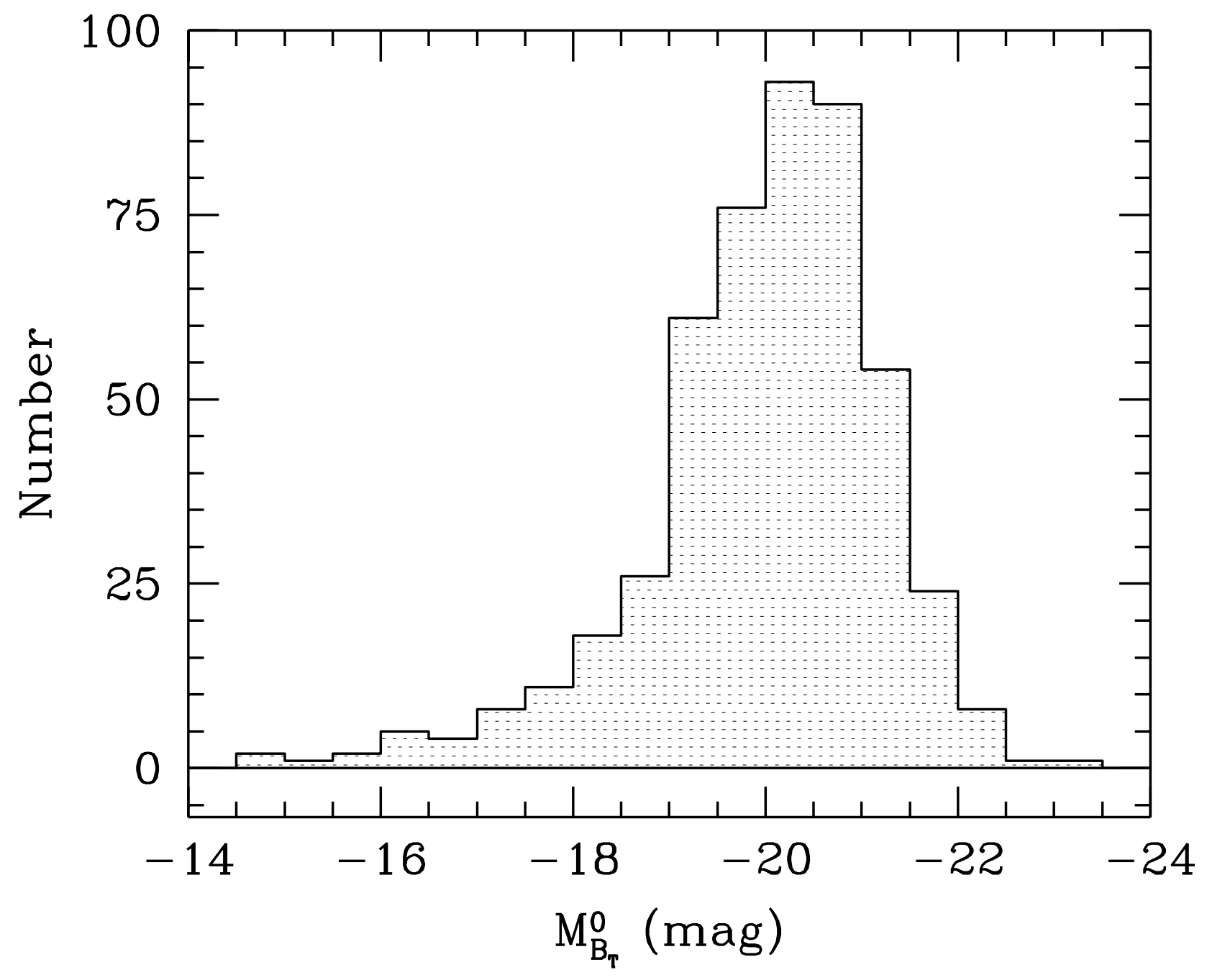




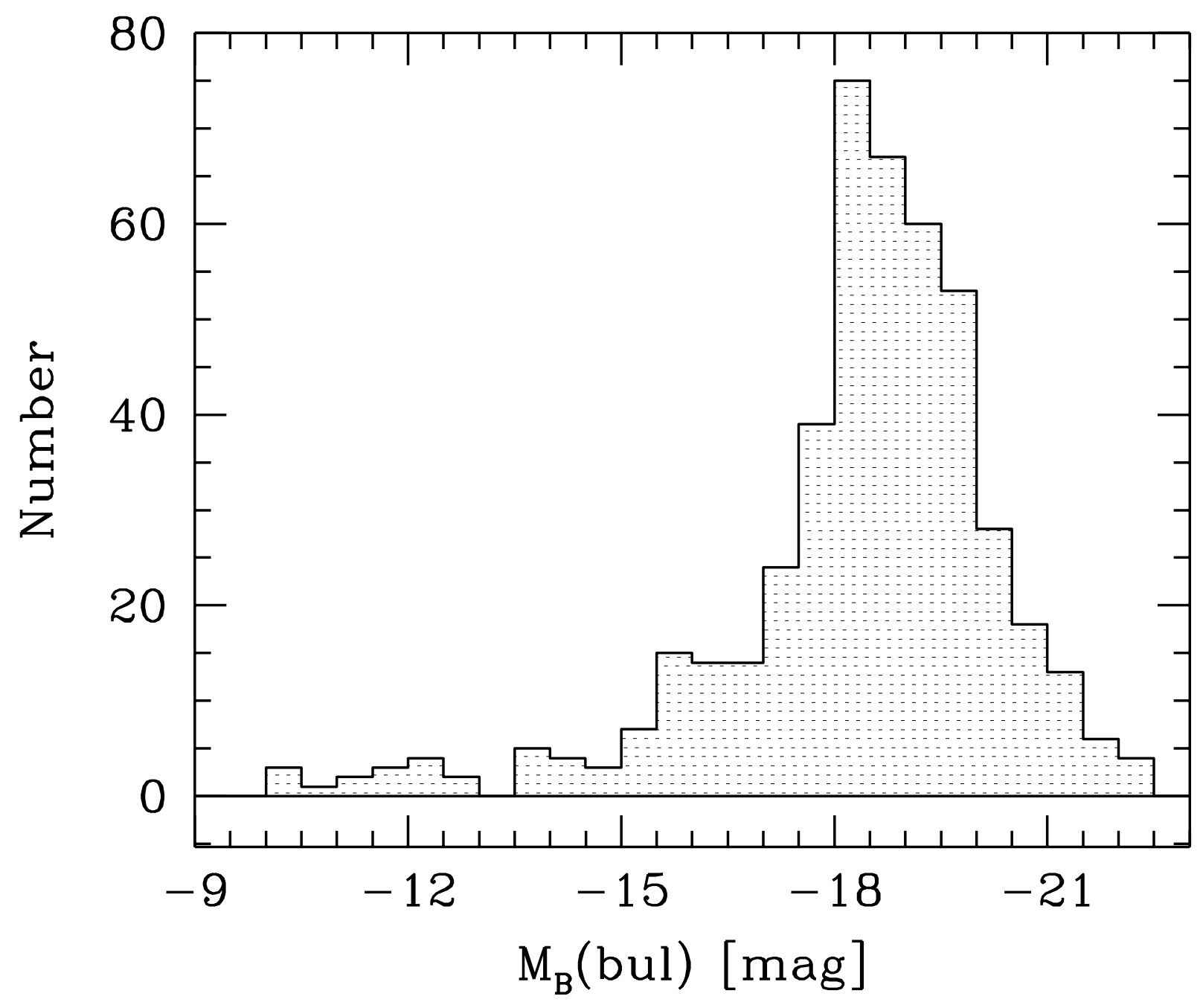




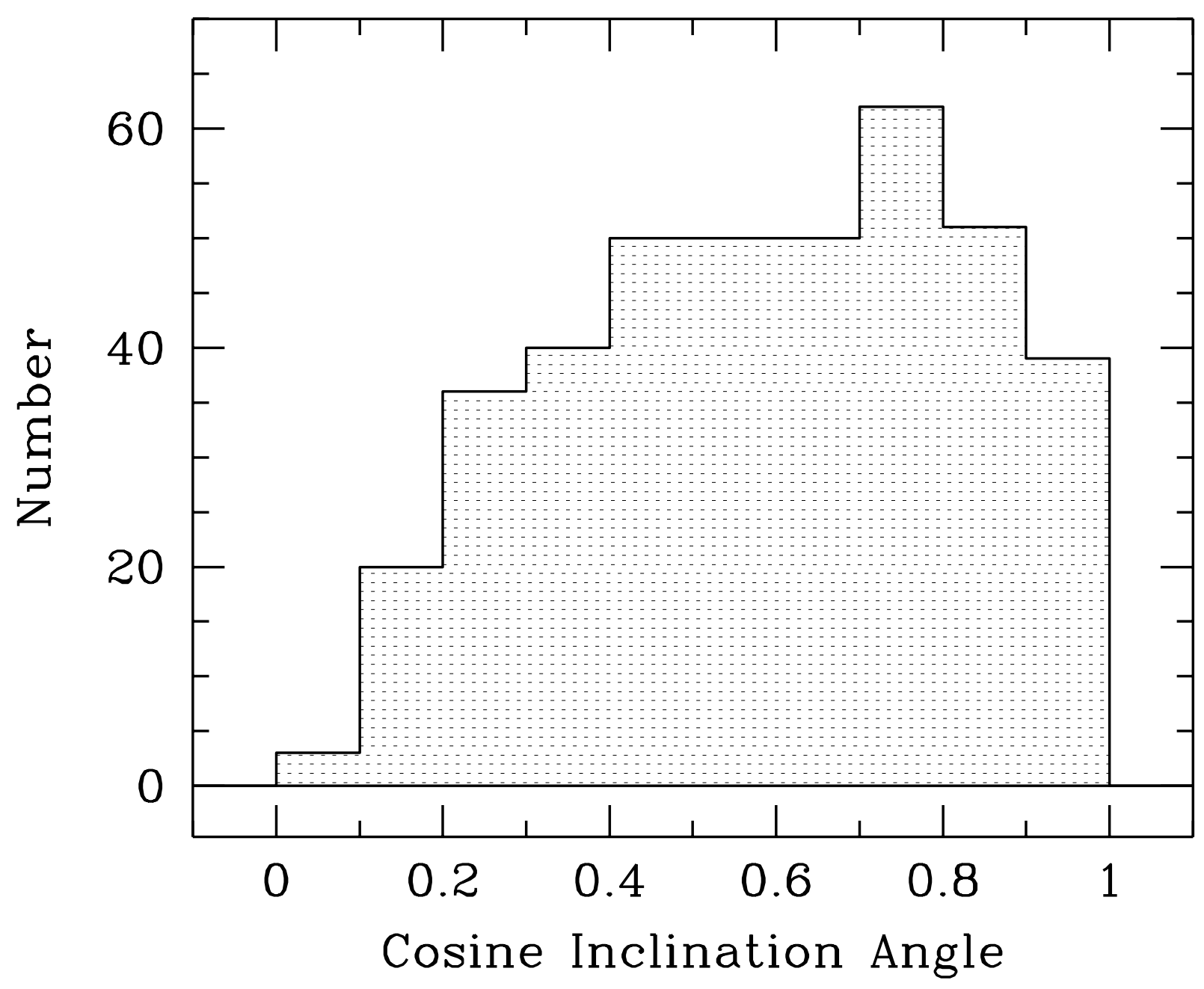


TABLE 1

LIST OF TEMPLATE GALAXIES

\begin{tabular}{cccccc}
\hline \hline Galaxy & Code & Galaxy & Code & Galaxy & Code \\
\hline NGC 205 & 1 & NGC 4026 & 28 & NGC 4570 & 55 \\
NGC 221 & 2 & NGC 4179 & 29 & NGC 4578 & 56 \\
NGC 224 & 3 & NGC 4251 & 30 & NGC 4608 & 57 \\
NGC 278 & $4^{\text {a }}$ & NGC 4262 & 31 & NGC 4612 & 58 \\
NGC 507 & 5 & NGC 4267 & 32 & NGC 4621 & 59 \\
NGC 628 & 6 & NGC 4291 & 33 & NGC 4648 & 60 \\
NGC 777 & $7^{\text {a }}$ & NGC 4324 & $34^{\mathrm{a}}$ & NGC 4638 & 61 \\
NGC 821 & 8 & NGC 4346 & $35^{\mathrm{a}}$ & NGC 4649 & 62 \\
NGC 890 & 9 & NGC 4339 & 36 & NGC 4660 & 63 \\
NGC 1023 & 10 & NGC 4340 & 37 & NGC 4665 & 64 \\
NGC 2300 & 11 & NGC 4350 & $38^{\mathrm{a}}$ & NGC 4754 & 65 \\
NGC 2549 & 12 & NGC 4365 & 39 & NGC 4762 & $66^{\mathrm{a}}$ \\
NGC 2634 & 13 & NGC 4371 & 40 & NGC 4914 & 67 \\
NGC 2775 & 14 & NGC 4379 & 41 & NGC 5308 & 68 \\
NGC 2950 & 15 & NGC 4382 & 42 & NGC 5473 & $69^{\mathrm{a}}$ \\
NGC 3115 & 16 & NGC 4406 & 43 & NGC 5557 & 70 \\
NGC 3193 & $17^{\mathrm{a}}$ & NGC 4414 & 44 & NGC 5576 & 71 \\
NGC 3377 & 18 & NGC 4417 & 45 & NGC 5638 & 72 \\
NGC 3384 & 19 & NGC 4421 & 46 & NGC 5668 & 73 \\
NGC 3412 & 20 & NGC 4442 & 47 & NGC 5831 & 74 \\
NGC 3521 & $21^{\mathrm{a}}$ & NGC 4461 & 48 & NGC 5982 & $75^{\mathrm{a}}$ \\
NGC 3608 & $22^{\mathrm{a}}$ & NGC 4472 & $49^{\mathrm{a}}$ & NGC 6654 & 76 \\
NGC 3610 & 23 & NGC 4473 & 50 & NGC 7332 & 77 \\
NGC 3613 & 24 & NGC 4478 & 51 & NGC 7457 & 78 \\
NGC 3640 & 25 & NGC 4494 & $52^{\mathrm{a}}$ & NGC 7619 & 79 \\
NGC 3838 & 26 & NGC 4503 & 53 & & \\
NGC 3900 & $27^{\mathrm{a}}$ & NGC 4564 & 54 & & \\
\hline & & & & & \\
\hline
\end{tabular}

${ }^{a}$ Only the blue spectrum used as template; the red spectrum contains weak emission lines. 
TABLE 13

SUMMARY OF THE MORPHOLOGICAL TYPES IN THE PALOMAR SURVEY

\begin{tabular}{|c|c|c|c|c|c|c|c|c|c|c|c|}
\hline \multirow[t]{2}{*}{ Hubble Type } & \multirow[t]{2}{*}{$\mathrm{T}$} & \multicolumn{2}{|c|}{ Total } & \multicolumn{2}{|c|}{ Unbarred } & \multicolumn{2}{|c|}{ Barred } & \multicolumn{2}{|c|}{ Weakly Barred } & \multicolumn{2}{|c|}{ Strongly Barred } \\
\hline & & No. & $\%$ & No. & $\%$ & No. & $\%$ & No. & $\%$ & No. & $\%$ \\
\hline $\mathrm{E}$ & -6 to -4 & 57 & 11.7 & 57 & 100.0 & 0 & 0.0 & 0 & 0.0 & 0 & 0.0 \\
\hline S0 & -3 to -1 & 88 & 18.1 & 51 & 57.9 & 37 & 42.1 & 8 & 9.1 & 29 & 33.0 \\
\hline $\mathrm{S} 0 / \mathrm{a}-\mathrm{Sab}$ & 0 to 2 & 77 & 15.8 & 34 & 44.2 & 43 & 55.8 & 19 & 24.7 & 24 & 31.1 \\
\hline $\mathrm{Sb}-\mathrm{Sbc}$ & 3 to 4 & 103 & 21.2 & 44 & 42.7 & 59 & 57.3 & 41 & 39.8 & 18 & 17.5 \\
\hline $\mathrm{Sc}-\mathrm{Scd}$ & 5 to 6 & 109 & 22.4 & 47 & 43.1 & 62 & 56.9 & 41 & 37.6 & 21 & 19.3 \\
\hline $\mathrm{Sd}-\mathrm{Sdm}$ & 7 to 8 & 19 & 3.9 & 5 & 26.3 & 14 & 73.7 & 5 & 26.3 & 9 & 47.4 \\
\hline $\mathrm{Sm}-\mathrm{Im}$ & 9 to 10 & 21 & 4.3 & 3 & 14.3 & 18 & 85.7 & 3 & 14.3 & 15 & 71.4 \\
\hline $\mathrm{I} 0$ & 90 & 5 & 1.0 & 5 & 100.0 & 0 & 0.0 & 0 & 0.0 & 0 & 0.0 \\
\hline $\mathrm{Pec}+\mathrm{S}$ pec & $99, \ldots$ & 7 & 1.4 & 6 & 85.7 & 1 & 14.3 & 1 & 14.3 & 0 & 0.0 \\
\hline All & & 486 & 100.0 & 252 & 51.9 & 234 & 48.1 & 118 & 24.3 & 116 & 23.8 \\
\hline
\end{tabular}

Note.- "Strongly barred" and "weakly barred galaxies refer to those classified in the RC3 system as "B" and "AB," respectively, while "barred" is the sum of $\mathrm{B}$ and $\mathrm{AB}$. 